\title{
Hydrodynamic modes for a granular gas from kinetic theory
}

\author{
J. Javier Brey \\ Física Teórica, Universidad de Sevilla, Apartado de Correos 1065, E-41080, Sevilla, Spain \\ James W. Dufty \\ Department of Physics, University of Florida, Gainesville, Florida 32611, USA
}

(Received 7 February 2005; published 18 July 2005)

\begin{abstract}
Small perturbations of the homogeneous cooling state for a low density granular gas are described by means of the linearized Boltzmann equation. The spectrum of the generator for this dynamics is shown to contain points corresponding to hydrodynamic excitations. The corresponding eigenvectors and eigenvalues are calculated to Navier-Stokes order and shown to agree with those obtained by the Chapman-Enskog method. The conditions for the hydrodynamic excitations to dominate all other excitations are discussed.
\end{abstract}

DOI: 10.1103/PhysRevE.72.011303

PACS number(s): 45.70. $-\mathrm{n}, 05.20 . \mathrm{Dd}, 51.10 .+\mathrm{y}$

\section{INTRODUCTION}

The use of hydrodynamic equations to describe granular fluids in rapid flow has been in practice for many years [1]. The justification for this fluidlike description and prediction of the transport coefficients appearing in these equations has been the focus of attention for some time as well [2-4]. In recent years, an accurate derivation of Navier-Stokes order hydrodynamics has been given from the Boltzmann equation for granular gases using an adaptation of the ChapmanEnskog method for normal gases $[4,5]$. The expressions for the transport coefficients as a function of the degree of inelasticity have been confirmed by both Monte Carlo and molecular dynamics simulation [6]. Successful application of these Navier-Stokes equations to a number of different states also supports their validity [7-9]. However, the context of the hydrodynamic equations remains uncertain. What are the relevant space and time scales? How much inelasticity can be described in this way?

Such questions can be addressed for gases using the Boltzmann kinetic equation to describe the complete dynamics for properties of interest. Then it can be asked under what conditions do the hydrodynamic excitations emerge as the dominant dynamics. The analysis of this problem for normal gases is quite complete and precise $[10,11]$. The objective here is to initiate a similar formulation of the problem for granular gases [12]. First, solutions to the Boltzmann equation are considered for states that deviate from spatial homogeneity only by small perturbations. The dynamics in this case is governed by the linear Boltzmann operator for spatially inhomogeneous states. The spectrum of this operator determines all possible excitations on all space and time scales, and for all degrees of inelasticity. The first problem is to identify points in this spectrum corresponding to hydrodynamics. This is one of the main results reported here. Both the hydrodynamic eigenvalues and eigenfunctions are calculated for long wavelength excitations corresponding to Navier-Stokes order hydrodynamics. Their agreement with the corresponding results from the Chapman-Enskog method is established.

Next, the issue of conditions for the dominance of the hydrodynamic excitations, or modes, is considered. This en- tails showing that the hydrodynamic eigenvalues are smaller than all other parts of the spectrum, such that there is a long enough time scale for the latter to decay relative to the hydrodynamic modes. There are two new difficulties for granular gases. First, the hydrodynamic eigenvalues cannot be made arbitrarily small since they do not all scale with the inverse wavelength of the perturbation, as for normal gases. Second, there is a new characteristic frequency, the cooling rate, in addition to the collision frequency to set the scale of the spectrum. The cooling rate can be relatively large or small depending on the inelasticity of the gas particle collisions.

Analysis of the nonhydrodynamic spectrum of the Boltzmann equation for a granular gas remains a difficult unsolved problem. Instead, we consider here a simpler kinetic model [13] that retains the exact hydrodynamic spectrum and allows identification of the entire spectrum as well. It is found that the time scale for the hydrodynamic excitations is longer than that for all other excitations, for any degree of inelasticity. A Brief Report of these results has been given in Ref. [12], with the details and elaboration given below.

The plan of the paper is as follows. In the next section, a short summary of the Boltzmann equation is given, and the Navier-Stokes equations derived from it by the ChapmanEnskog procedure are recalled. More details are given in Appendix A. Also, the hydrodynamic equations are linearized and the hydrodynamic modes to second order in the wave vector are identified.

In Sec. III, the structure of the linear Boltzmann equation is discussed, emphasizing the relevance of the spectrum of the operator generating the linear dynamics to determine the existence and validity of hydrodynamics. The first of the above issues is addressed in Sec. IV, where the kinetic theory hydrodynamic modes are identified in the long wavelength limit. A technical point associated with the non Hermitian character of the operator is discussed in Appendix B. The results are extended to Navier-stokes order in Sec. V, and the obtained expressions are shown to agree with those derived in Sec. II from the hydrodynamic equations. Details of the calculations are given in Appendix C. The possibility of a description in terms of the hydrodynamic modes is studied in Sec. VI, by means of a model kinetic equation. It is estab- 
lished that there are length and time scales on which only the excitations associated to the hydrodynamic modes persist. The mathematical details are given in Appendix D. Finally, the last section contains a short summary of the main points and conclusions in the paper.

\section{BOLTZMANN EQUATION AND HYDRODYNAMIC MODES}

In all of the following, the simplest model of a granular fluid is considered: a low density gas of smooth and inelastic hard spheres $(d=3)$ or disks $(d=2)$ of mass $m$ and diameter $\sigma$. The binary collision rule given below is characterized by a constant coefficient of restitution $\alpha$, defined in the interval $0<\alpha \leqslant 1$, and measuring the loss of energy in each collision. At sufficiently low density, the distribution function $f(\boldsymbol{r}, \boldsymbol{v}, t)$ is determined from the Boltzmann equation $[4,14,15]$

$$
\left(\partial_{t}+\boldsymbol{v} \cdot \boldsymbol{\nabla}\right) f=J[f, f],
$$

where $J$ is the inelastic Boltzmann collision operator defined by

$$
\begin{aligned}
J[X, Y] \equiv & \sigma^{d-1} \int d \boldsymbol{v}_{1} \int d \hat{\boldsymbol{\sigma}} \Theta(\hat{\boldsymbol{\sigma}} \cdot \boldsymbol{g}) \hat{\boldsymbol{\sigma}} \cdot \boldsymbol{g} \\
& \times\left[\alpha^{-2} X\left(\boldsymbol{r}, \boldsymbol{v}^{\prime}, t,\right) Y\left(\boldsymbol{r}, \boldsymbol{v}_{1}^{\prime}, t\right)-X(\boldsymbol{r}, \boldsymbol{v}, t) Y\left(\boldsymbol{r}, \boldsymbol{v}_{1}, t\right)\right],
\end{aligned}
$$

for arbitrary functions $X(\boldsymbol{v})$ and $Y(\boldsymbol{v})$. Here, $\hat{\boldsymbol{\sigma}}$ is a unit vector along the line joining the centers of the colliding pair, $\Theta$ is the Heaviside step function, and $\boldsymbol{g}=\boldsymbol{v}-\boldsymbol{v}_{1}$. The primes on the velocities denote the initial values $\left\{\boldsymbol{v}_{1}^{\prime}, \boldsymbol{v}_{2}^{\prime}\right\}$ that lead to $\left\{\boldsymbol{v}_{1}, \boldsymbol{v}_{2}\right\}$ following a "restituting" binary collision

$\boldsymbol{v}^{\prime}=\boldsymbol{v}-\frac{1}{2}\left(1+\alpha^{-1}\right)(\hat{\boldsymbol{\sigma}} \cdot \boldsymbol{g}) \hat{\boldsymbol{\sigma}}, \quad \boldsymbol{v}_{1}^{\prime}=\boldsymbol{v}_{1}+\frac{1}{2}\left(1+\alpha^{-1}\right)(\hat{\boldsymbol{\sigma}} \cdot \boldsymbol{g}) \hat{\boldsymbol{\sigma}}$.

The usual Boltmann collision operator is recovered from Eq.

(2) in the elastic limit $\alpha=1$.

The macroscopic variables of interest are the hydrodynamic fields, i.e., the density $n(\boldsymbol{r}, t)$, the flow velocity $\boldsymbol{u}(\boldsymbol{r}, t)$, and the (granular) temperature $T(\boldsymbol{r}, t)$. They are defined as moments of the solution to the Boltzmann equation

$$
\left(\begin{array}{c}
n(\boldsymbol{r}, t) \\
n(\boldsymbol{r}, t) \boldsymbol{u}(\boldsymbol{r}, t) \\
\frac{d}{2} n(\boldsymbol{r}, t) T(\boldsymbol{r}, t)
\end{array}\right)=\int d \boldsymbol{v}\left(\begin{array}{c}
1 \\
\boldsymbol{v} \\
\frac{1}{2} m(\boldsymbol{v}-\boldsymbol{u})^{2}
\end{array}\right) f(\boldsymbol{r}, \boldsymbol{v}, t) .
$$

An exact set of equations for these variables is obtained from the following properties of the collision operator

$$
\int d \boldsymbol{v}\left(\begin{array}{c}
1 \\
\boldsymbol{v} \\
\frac{1}{2} m(\boldsymbol{v}-\boldsymbol{u})^{2}
\end{array}\right) J[f, f]=\left(\begin{array}{c}
0 \\
0 \\
-\frac{d}{2} n T \zeta
\end{array}\right) .
$$

The first two equations follow from conservation of mass and momentum in the particle collisions. The last equation reflects the loss of energy in collisions due to the inelasticity. This appears through the "cooling rate" $\zeta(\boldsymbol{r}, t) \geqslant 0$, defined by this equation.

The macroscopic balance equations resulting from the above properties are

$$
\begin{gathered}
\partial_{t} n+\boldsymbol{\nabla} \cdot(n \boldsymbol{u})=0 \\
\left(\partial_{t}+\boldsymbol{u} \cdot \boldsymbol{\nabla}\right) \boldsymbol{u}+(m n)^{-1}[\boldsymbol{\nabla}(n T)+\boldsymbol{\nabla} \cdot \Pi]=0, \\
\left(\partial_{t}+\boldsymbol{u} \cdot \boldsymbol{\nabla}+\zeta\right) T+\frac{2}{n d}(n T \boldsymbol{\nabla} \cdot \boldsymbol{u}+\Pi: \boldsymbol{\nabla} \mathbf{u}+\boldsymbol{\nabla} \cdot \boldsymbol{q})=0 .
\end{gathered}
$$

The functionals giving the dissipative part of the pressure tensor $\Pi$ and the heat flux $\boldsymbol{q}$ are also moments of the solution to the Boltzmann equation

$$
\begin{gathered}
\Pi(\mathbf{r}, t)=\int d \boldsymbol{v} m\left(\boldsymbol{V} \boldsymbol{V}-\frac{V^{2}}{d} \mathrm{I}\right) f(\boldsymbol{r}, \boldsymbol{v}, t), \\
\boldsymbol{q}(\boldsymbol{r}, t)=\int d \boldsymbol{v}\left(\frac{m}{2} V^{2}-\frac{d+2}{2} T\right) \boldsymbol{V} f(\boldsymbol{r}, \boldsymbol{v}, t),
\end{gathered}
$$

where I is the unit tensor of dimension $d$ and $\boldsymbol{V} \equiv \boldsymbol{v}-\boldsymbol{u}$ the so-called peculiar velocity. Equations (6)-(8) are the basis for a hydrodynamic description, once $\Pi(\boldsymbol{r}, t), \boldsymbol{q}(\boldsymbol{r}, t)$, and $\zeta(\boldsymbol{r}, t)$ are specified. These can be obtained from their definitions using a solution of the Boltzmann equation generated by the Chapman-Enskog method. This method assumes the existence of a solution whose space and time dependence is given entirely through the hydrodynamic fields and their gradients (i.e., a "normal" solution). As a result, $\Pi(\boldsymbol{r}, t)$ and $\boldsymbol{q}(\boldsymbol{r}, t)$ are given in terms of these variables, and Eqs. (6)-(8) become a closed set of hydrodynamic equations. The primary results of this method are recalled in Appendix A. To leading order in the spatial gradients, the dissipative fluxes are found to be given by $[5,6]$

$$
\begin{gathered}
\Pi_{i j}(\boldsymbol{r}, t)=-\eta\left(\boldsymbol{\nabla}_{i} u_{j}+\boldsymbol{\nabla}_{j} u_{i}-\frac{2}{d} \delta_{i j} \boldsymbol{\nabla} \cdot \boldsymbol{u}\right), \\
\boldsymbol{q}(\boldsymbol{r}, t)=-\kappa \boldsymbol{\nabla} T-\mu \boldsymbol{\nabla} n .
\end{gathered}
$$

For the cooling rate the result is

$$
\begin{aligned}
\zeta(\boldsymbol{r}, t)= & n \sigma^{d-1}\left(\frac{2 T}{m}\right)^{1 / 2} \zeta_{0}^{*}+\zeta_{1} \nabla^{2} T+\zeta_{2} \nabla^{2} n \\
& + \text { bilinear in } \boldsymbol{\nabla} n, \boldsymbol{\nabla} T, \boldsymbol{\nabla} \boldsymbol{u} \text { terms },
\end{aligned}
$$

with $\zeta_{0}^{*}$ being a dimensionless positive constant proportional to $\left(1-\alpha^{2}\right)$. The explicit forms for the shear viscosity $\eta$, the thermal conductivity $\kappa$, and the new transport coefficients $\mu, \zeta_{1}$, and $\zeta_{2}$, peculiar to granular gases, are given in Appendix A. The nonlinear contributions to $\zeta(t)$ indicated in Eq. (13) play no role in the following linear analysis and will be not discussed further in this paper.

Equations (6)-(8) together with the "constitutive relations" (11)-(13) are the Navier-Stokes hydrodynamic equa- 
tions for a granular gas. A special solution of these equations is that for a homogeneous state, characterized by a constant density $n_{\mathrm{HCS}}$, a vanishing velocity flow $\boldsymbol{u}_{\mathrm{HCS}}=0$, and a uniform temperature $T_{\mathrm{HCS}}(t)$ determined from

$$
\partial_{t} T_{\mathrm{HCS}}(t)=-n_{\mathrm{HCS}} \sigma^{d-1}\left(\frac{2}{m}\right)^{1 / 2} \zeta_{0}^{*} T_{\mathrm{HCS}}^{3 / 2}(t) .
$$

This is referred to as the homogeneous cooling state (HCS), since the only macroscopic dynamics is the monotonic decrease in the temperature field. It is easily seen that the HCS as defined above is also a solution of the exact balance equations, taking into account that $\zeta_{\mathrm{HCS}} \propto n_{\mathrm{HCS}} \sigma^{d-1}\left(T_{\mathrm{HCS}} / m\right)^{1 / 2}$ on dimensional grounds. In the following, the solution to the Boltzmann equation will be considered for small perturbations of the HCS, and it will be useful to have the corresponding results from Navier-Stokes hydrodynamics. The linearized Navier-Stokes equations have time dependent coefficients, since the distribution function of the reference HCS depends on time. This complication can be overcome by the introduction of suitable dimensionless space and time scales, as well as scaled hydrodynamic fields. Thus we define $r^{*}$ and $s$ by

$$
r^{*}=\frac{\boldsymbol{r}}{\ell}, \quad d s=\frac{v_{\mathrm{HCS}}(t)}{\ell} d t .
$$

Here, $v_{\mathrm{HCS}}(t) \equiv\left[2 T_{\mathrm{HCS}}(t) / m\right]^{1 / 2}$ is the thermal velocity in the HCS, and $\ell \equiv\left(n_{\mathrm{HCS}} \sigma^{d-1}\right)^{-1}$ is proportional to the mean free path of the particles. The dimensionless fields $\delta y_{j}\left(\boldsymbol{r}^{*}, s\right)$ are chosen as

$$
\begin{aligned}
& \rho\left(\boldsymbol{r}^{*}, s\right)= \frac{n(\boldsymbol{r}, t)-n_{\mathrm{HCS}}}{n_{\mathrm{HCS}}}, \quad \theta\left(\boldsymbol{r}^{*}, s\right)=\frac{T(\boldsymbol{r}, t)-T_{\mathrm{HCS}}(t)}{T_{\mathrm{HCS}}(t)}, \\
& \boldsymbol{\omega}\left(\boldsymbol{r}^{*}, s\right)=\frac{\boldsymbol{u}(\boldsymbol{r}, t)}{v_{\mathrm{HCS}}(t)} .
\end{aligned}
$$

In addition, since the equations are linear and the reference state is homogeneous, it is sufficient to consider a single Fourier mode

$$
\delta y_{j}\left(\boldsymbol{r}^{*}, s\right)=e^{i \boldsymbol{k} \cdot \boldsymbol{r}^{*}} \delta \tilde{y}_{j}(\boldsymbol{k}, s) .
$$

The velocity components are chosen as a longitudinal component relative to $\boldsymbol{k}, \widetilde{\boldsymbol{\omega}}_{\|}(\boldsymbol{k}, s)=\hat{\boldsymbol{k}} \cdot \widetilde{\boldsymbol{\omega}}(\boldsymbol{k}, s)$, and $d-1$ transverse components $\quad \widetilde{\boldsymbol{\omega}}_{\perp, i}(\boldsymbol{k}, s)=\hat{\boldsymbol{e}}^{i} \cdot \widetilde{\boldsymbol{\omega}}(\boldsymbol{k}, s)$, where $\left\{\hat{\boldsymbol{k}} \equiv \boldsymbol{k} / k, \hat{\boldsymbol{e}}^{(i)}, i\right.$ $=1, \ldots, d-1\}$ are a set of $d$ pairwise orthogonal unit vectors . The dimensionless linear Navier-Stokes equations then become a system of ordinary differential equations with constant coefficients that can be expressed in the compact form [5]

$$
\partial_{s} \delta \widetilde{\boldsymbol{y}}(\boldsymbol{k}, s)+\mathrm{K}(k) \cdot \delta \widetilde{\boldsymbol{y}}(\boldsymbol{k}, s)=0,
$$

where we use a $(d+2)$ dimensional space representation with $\delta \widetilde{y}(\boldsymbol{k}, s)$ being the vector

$$
\delta \widetilde{\mathbf{y}}(\boldsymbol{k}, s)=\left(\begin{array}{c}
\widetilde{\rho}(\boldsymbol{k}, s) \\
\widetilde{\theta}(\boldsymbol{k}, s) \\
\widetilde{\boldsymbol{\omega}}_{\|}(\boldsymbol{k}, s) \\
\widetilde{\boldsymbol{\omega}}_{\perp}(\boldsymbol{k}, s)
\end{array}\right),
$$

and $\widetilde{\boldsymbol{\omega}}_{\perp}(\boldsymbol{k}, s)$ denoting the vector formed by the $d-1 \mathrm{com}-$ ponents $\widetilde{\omega}_{\perp, i}(\boldsymbol{k}, s)$. The matrix $\mathrm{K}(k)$ is block diagonal, expressing the decoupling of transverse and longitudinal modes

$$
\mathrm{K}(k)=\left(\begin{array}{cc}
\mathrm{K}_{1} & 0 \\
0 & \mathrm{~K}_{2}
\end{array}\right),
$$

$$
\mathrm{K}_{1}(k)=\left(\begin{array}{ccc}
0 & 0 & i k \\
\zeta_{0}^{*}+\left(\mu^{*}-\zeta_{2}^{*}\right) k^{2} & \frac{\zeta_{0}^{*}}{2}+\left(\kappa^{*}-\zeta_{1}^{*}\right) k^{2} & \frac{2 i}{d} k \\
\frac{i}{2} k & \frac{i}{2} k & -\frac{\zeta_{0}^{*}}{2}+\frac{2(d-1)}{d} \eta^{*} k^{2}
\end{array}\right)
$$

$$
\mathrm{K}_{2}(k)=-\left(\frac{\zeta_{0}^{*}}{2}-\eta^{*} k^{2}\right) \mathrm{I}
$$

In the above expressions, $\mathrm{K}_{2}$ and $\mathrm{I}$ are matrices of dimension $d-1$. The dimensionless transport coefficients used in the above equations are defined by

$$
\eta^{*}=\frac{\eta}{\ell m n_{\mathrm{HCS}} v_{\mathrm{HCS}}}, \quad \kappa^{*}=\frac{2 \kappa}{d \ell n_{\mathrm{HCS}} v_{\mathrm{HCS}}}, \quad \mu^{*}=\frac{2 \mu}{d \ell T_{\mathrm{HCS}} v_{\mathrm{HCS}}},
$$

$$
\zeta_{1}^{*}=\frac{T_{\mathrm{HCS}} \zeta_{1}}{\ell v_{\mathrm{HCS}}}, \quad \zeta_{2}^{*}=\frac{n_{\mathrm{HCS} \zeta_{2}}}{\ell v_{\mathrm{HCS}}}
$$

The formal solution to the initial value problem (18) is

$$
\delta \widetilde{y}(\boldsymbol{k}, s)=e^{-\mathrm{K}(k) s} \cdot \delta \widetilde{y}(\boldsymbol{k}, 0) .
$$

The eigenvalues and eigenvectors of the generator $\mathrm{K}$ for this dynamics define the $d+2$ Navier-Stokes order hydrodynamic modes. They are given by the solutions of the equation 


$$
\mathrm{K}(k) \cdot \boldsymbol{\varphi}_{j}(\boldsymbol{k})=\lambda_{j}(k) \boldsymbol{\varphi}_{j}(\boldsymbol{k}), \quad j=1, \ldots, d+2 .
$$

A simple calculation provides the expressions for the eigenvalues to order $k^{2}$. They are given by

$$
\begin{gathered}
\lambda_{1}(k)=\frac{k^{2}}{\zeta_{0}^{*}}, \quad \lambda_{2}(k)=\frac{\zeta_{0}^{*}}{2}-\left(\frac{d+1}{d \zeta_{0}^{*}}-\kappa^{*}+\zeta_{1}^{*}\right) k^{2}, \\
\lambda_{\|}(k)=-\frac{\zeta_{0}^{*}}{2}+\left[\frac{1}{d \zeta_{0}^{*}}+\frac{2(d-1) \eta^{*}}{d}\right] k^{2}, \quad \lambda_{\perp}(k)=-\frac{\zeta_{0}^{*}}{2}+\eta^{*} k^{2},
\end{gathered}
$$

the eigenvalue $\lambda_{\perp}(k)$ being $(d-1)$-fold degenerate. The corresponding eigenvectors to leading order in $k$ are

$$
\begin{array}{cc}
\boldsymbol{\varphi}_{1}(\boldsymbol{k})=\left(\begin{array}{c}
1 \\
-2 \\
0 \\
\mathbf{0}
\end{array}\right), \quad \boldsymbol{\varphi}_{2}(\boldsymbol{k})=\left(\begin{array}{l}
0 \\
1 \\
0 \\
\mathbf{0}
\end{array}\right), \quad \boldsymbol{\varphi}_{\|}(\boldsymbol{k})=\left(\begin{array}{l}
0 \\
0 \\
1 \\
\mathbf{0}
\end{array}\right), \\
\boldsymbol{\varphi}_{\perp, i}(\boldsymbol{k})=\left(\begin{array}{l}
0 \\
0 \\
0 \\
\hat{\boldsymbol{i}}
\end{array}\right) .
\end{array}
$$

Here, $\mathbf{0}=0$, and $\hat{\boldsymbol{i}}=\hat{\mathbf{1}}=1$, for $d=2, \mathbf{0}=\left(\begin{array}{l}0 \\ 0\end{array}\right), \hat{\mathbf{1}}=\left(\begin{array}{l}1 \\ 0\end{array}\right)$, and $\hat{\mathbf{2}}=\left(\begin{array}{l}0 \\ 1\end{array}\right)$, for $d=3$.

The first of these modes is excited by the condition $\widetilde{\theta}(\boldsymbol{k}, 0)=-2 \widetilde{\rho}(\boldsymbol{k}, 0)$ at zero flow velocity. The second is produced by a temperature perturbation at constant density and also zero velocity, while the third one corresponds to a longitudinal velocity perturbation at constant temperature and density. There is a $(d-1)$-fold degeneracy for the shear modes of eigenvalue $\lambda_{\perp}$. These diffusive modes are excited by a perturbation of the velocity field in the transverse plane orthogonal to $\boldsymbol{k}$.

It should be noted that while the above analysis is restricted to the Navier-Stokes equations, derived from the Boltzmann equation by the Chapman-Enskog method, the eigenvalues and eigenvectors to order $k$ follow more generally from the exact macroscopic balance equations and do not depend on the approximate constitutive equations (11) and (12). The hydrodynamic modes sought by kinetic theory in the subsequent sections can therefore be defined as those excitations due to small perturbations which agree with the above in the long wavelength limit. Analyticity then allows extension of that identification to shorter wavelengths. A consistency check of the Chapman-Enskog method is agreement with the above results at order $k^{2}$. However, the concept of hydrodynamic modes in this context does not require the validity of the Chapman-Enskog method nor the limitation to the Navier-Stokes approximation.

\section{LINEAR BOLTZMANN EQUATION}

A more complete and accurate description of the response to small spatial perturbations of the density, temperature, and flow velocity is obtained directly from the Boltzmann equation. Consider first an isolated system. As already indicated in the previous section, the exact balance equations have a solution describing the HCS, with a monotonically decreasing temperature obeying Eq. (14). The solution to the Boltzmann equation corresponding to this macroscopic state is characterized by the scaling form [4]

$$
f_{\mathrm{HCS}}(\boldsymbol{v}, t)=n_{\mathrm{HCS}} v_{\mathrm{HCS}}^{-d}(t) \phi\left(V^{*}\right),
$$

with

$$
\boldsymbol{V}^{*}=\frac{\boldsymbol{V}}{v_{\mathrm{HCS}}(t)}=\frac{\boldsymbol{v}-\boldsymbol{u}_{\mathrm{HCS}}}{v_{\mathrm{HCS}}(t)} .
$$

Substitution into the Boltzmann equation gives

$$
\frac{\zeta_{\mathrm{HCS}}}{2} \frac{\partial}{\partial \boldsymbol{V}} \cdot\left(\boldsymbol{V} f_{\mathrm{HCS}}\right)=J\left[f_{\mathrm{HCS}}, f_{\mathrm{HCS}}\right] \text {. }
$$

For later convenience, a constant velocity $\boldsymbol{u}_{\mathrm{HCS}}$ for the system as a whole has been included, although this can always be removed by means of a Gallilean transformation. Then, in the following it will be considered that $\boldsymbol{u}_{\mathrm{HCS}}=\mathbf{0}$, unless it be explicitly established otherwise. An exact and explicit solution of Eq. (31) is not known yet, but the behavior of $\phi$ at large and small velocities has been determined [14] and the results obtained by the direct simulation Monte Carlo method strongly supports the existence of such a scaling form [16].

The HCS distribution function is a "universal" homogeneous solution in the same sense as the Maxwellian for elastic collisions. An arbitrary homogeneous state is expected to approach the HCS after a few collisions. Therefore, in discussing response of any homogeneous state to small spatial perturbations, it is sufficient to consider the HCS as the reference state. All the other cases will simply induce additional short time transients.

Consider then small spatial perturbations of the HCS

$$
f(\boldsymbol{r}, \boldsymbol{v}, t)=f_{\mathrm{HCS}}(\boldsymbol{v}, t)[1+\Delta(\boldsymbol{r}, \boldsymbol{v}, t)],|\Delta(\boldsymbol{r}, \boldsymbol{v}, t)| \ll 1 .
$$

To linear order in $\Delta(\boldsymbol{r}, \boldsymbol{v}, t)$, the Boltzmann equation becomes

$$
\left[\partial_{t}+\boldsymbol{v} \cdot \boldsymbol{\nabla}+L(t)\right]\left(f_{\mathrm{HCS}} \Delta\right)=0,
$$

where $L(t)$ is the linearized Boltzmann collision operator given by

$$
L(t) X(\boldsymbol{v})=-J\left[f_{\mathrm{HCS}}, X\right]-J\left[X, f_{\mathrm{HCS}}\right],
$$

for arbitrary $X(\boldsymbol{v})$. Just as for the analysis of the NavierStokes equations in the previous section, the above linear kinetic equation takes a simpler form when expressed in terms of the dimensionless variables defined in Eq. (15), and considering a single Fourier mode

$$
\Delta(\boldsymbol{r}, \boldsymbol{v}, t)=e^{i \boldsymbol{k} \cdot \boldsymbol{r}^{*}} \widetilde{\Delta}\left(\boldsymbol{k}, \boldsymbol{v}^{*}, s\right),
$$

where $\boldsymbol{v}^{*}=\boldsymbol{v} / v_{\mathrm{HCS}}(t)$. Then, Eq. (33) becomes

$$
\left(\partial_{s}+i \boldsymbol{k} \cdot \boldsymbol{v}^{*}+\mathcal{L}^{*}\right) \widetilde{\Delta}\left(\boldsymbol{k}, \boldsymbol{v}^{*}, s\right)=0 .
$$

The dimensionless operator $\mathcal{L}^{*}$ is now time independent and it is given by 


$$
\mathcal{L}^{*} X \equiv \frac{\zeta_{0}^{*}}{2} \phi^{-1} \frac{\partial}{\partial \boldsymbol{v}^{*}} \cdot\left(\boldsymbol{v}^{*} \phi X\right)+L^{*} X,
$$

where $L^{*}$ is the dimensionless linear Boltzmann collision operator

$$
\begin{aligned}
& L^{*} X \equiv-\phi^{-1}\left(J^{*}[\phi, \phi X]+J^{*}[\phi X, \phi]\right) \\
J^{*}[X, Y] \equiv & \int d \boldsymbol{v}_{1}^{*} \int d \hat{\boldsymbol{\sigma}} \Theta\left(\hat{\boldsymbol{\sigma}} \cdot \boldsymbol{g}^{*}\right) \hat{\boldsymbol{\sigma}} \cdot \boldsymbol{g}^{*}\left[\alpha^{-2} X\left(\boldsymbol{v}^{* \prime}\right) Y\left(\boldsymbol{v}_{1}^{* \prime}\right)\right. \\
& \left.-X\left(\boldsymbol{v}^{*}\right) Y\left(\boldsymbol{v}_{1}^{*}\right)\right] .
\end{aligned}
$$

Here, $\boldsymbol{v}^{* \prime}$ and $\boldsymbol{v}_{1}^{* \prime}$ are related with $\boldsymbol{v}^{*}$ and $\boldsymbol{v}_{1}^{*}$ by Eqs. (3). The operator $\mathcal{L}^{*}$ differs from the linearized Boltzmann collision operator $L^{*}$ by terms representing the cooling effects of the inelastic collisions. The latter arise because the derivative with respect to $s$ is taken at constant $\boldsymbol{v}^{*}$ rather than $\boldsymbol{v}$.

Solutions to the dimensionless, linear kinetic equation (36) for $\widetilde{\Delta}$ are sought in a Hilbert space whose scalar product is defined by

$$
(X, Y)=\int d \boldsymbol{v}^{*} \phi\left(v^{*}\right) X^{\dagger}\left(\boldsymbol{v}^{*}\right) Y\left(\boldsymbol{v}^{*}\right),
$$

with the dagger denoting complex conjugation. The formal solution to the kinetic equation is

$$
\begin{gathered}
\widetilde{\Delta}\left(\boldsymbol{k}, \boldsymbol{v}^{*}, s\right)=\frac{1}{2 \pi i} \oint d z e^{-z s} \mathcal{R}(z) \Delta^{*}\left(\boldsymbol{k}, \boldsymbol{v}^{*}, 0\right), \\
\mathcal{R}(z) \equiv\left(z-i \boldsymbol{k} \cdot \boldsymbol{v}^{*}-\mathcal{L}^{*}\right)^{-1},
\end{gathered}
$$

where the contour encloses the entire spectrum of $i \boldsymbol{k} \cdot \boldsymbol{v}^{*}$ $+\mathcal{L}^{*}$, both point and residual, counterclockwise. It is important to realize that all the linear excitations of the granular gas are determined from this spectrum. This formulation of the problem for small spatial perturbations provides a precise context for addressing many questions regarding hydrodynamics for a granular gas. The existence of the hydrodynamic modes and their role relative to other dynamical processes are determined by the characterization of the above spectrum. To see how this occurs, it is useful to recall briefly the status of the corresponding problem for gases with elastic collisions $[10,11]$. In that case, it has been proved that the hydrodynamic modes exist as $d+2$ poles located at the origin in the long wavelength limit and corresponding to the local conserved quantities. Furthermore, the remainder of the spectrum is bounded away from these poles, and the spectrum is analytic in $\boldsymbol{k}$ about $\boldsymbol{k}=0$, so this isolation of the hydrodynamic modes is preserved at finite wavelengths.

\section{EXISTENCE OF HYDRODYNAMIC MODES}

The spectrum of $i \boldsymbol{k} \cdot \boldsymbol{v}^{*}+\mathcal{L}^{*}$ is expected to be quite complex, based on the special case of elastic collisions, with points spectra, continua, and limit points. The hydrodynamic excitations, whenever they exist, are part of the point spectrum so in order to investigate them it suffices to consider the eigenvalue problem

$$
\left(i \boldsymbol{k} \cdot \boldsymbol{v}^{*}+\mathcal{L}^{*}\right) \Psi_{i}=\lambda_{i}(k) \Psi_{i}
$$

The search for hydrodynamic excitations can be carried out by assuming they are analytic in $k$ and looking first for the $\boldsymbol{k}=\mathbf{0}$ solutions of Eq. (43). The practical issue of constructing these modes at finite $k$ is addressed in the next section.

The central idea for constructing the hydrodynamic eigenvalues and eigenvectors at $k=0$ is the note that the HCS is parametrized by the hydrodynamic fields $n_{\mathrm{HCS}}, T_{\mathrm{HCS}}$, and $\boldsymbol{u}_{\mathrm{HCS}}$, which is now considered different from zero, as discussed at the beginning of the previous section. Therefore, differentiating the Boltzmann equation for the distribution function of the HCS, Eq. (31), with respect to these fields gives exact properties of the linearized Boltzmann collision operator. For example,

$$
\frac{\partial}{\partial n_{\mathrm{HCS}}}\left\{\frac{\zeta_{\mathrm{HCS}}}{2} \frac{\partial}{\partial \boldsymbol{V}} \cdot\left(\boldsymbol{V} f_{\mathrm{HCS}}\right)-J\left[f_{\mathrm{HCS}}, f_{\mathrm{HCS}}\right]\right\}=0
$$

gives directly

$$
\frac{1}{2} \frac{\partial \zeta_{\mathrm{HCS}}}{\partial n_{\mathrm{HCS}}} \frac{\partial}{\partial \boldsymbol{V}} \cdot\left(\boldsymbol{V} f_{\mathrm{HCS}}\right)+\frac{\zeta_{\mathrm{HCS}}}{2} \frac{\partial}{\partial \boldsymbol{V}} \cdot\left(\boldsymbol{V} \frac{\partial f_{\mathrm{HCS}}}{\partial n_{\mathrm{HCS}}}\right)+L \frac{\partial f_{\mathrm{HCS}}}{\partial n_{\mathrm{HCS}}}=0 .
$$

The derivatives of $\zeta_{\mathrm{HCS}}$ and $f_{\mathrm{HCS}}$ are easily calculated from the properties $\zeta_{\mathrm{HCS}} \propto n_{\mathrm{HCS}}$ and $f_{\mathrm{HCS}} \propto n_{\mathrm{HCS}}$. In terms of the dimensionless variables, and setting $\boldsymbol{u}_{\mathrm{HCS}}=\mathbf{0}$, Eq. (45) becomes

$$
\mathcal{L}^{*} 1=-\frac{\zeta_{0}^{*}}{2} \phi^{-1} \frac{\partial}{\partial \boldsymbol{v}^{*}} \cdot\left(\boldsymbol{v}^{*} \phi\right) .
$$

Next, calculate the derivative of Eq. (31) with respect to $T_{\mathrm{HCS}}$,

$$
\frac{1}{2} \frac{\partial \zeta_{\mathrm{HCS}}}{\partial T_{\mathrm{HCS}}} \frac{\partial}{\partial \boldsymbol{V}} \cdot\left(\boldsymbol{V} f_{\mathrm{HCS}}\right)+\frac{\zeta_{\mathrm{HCS}}}{2} \frac{\partial}{\partial \boldsymbol{V}} \cdot\left(\boldsymbol{V} \frac{\partial f_{\mathrm{HCS}}}{\partial T_{\mathrm{HCS}}}\right)+L \frac{\partial f_{\mathrm{HCS}}}{\partial T_{\mathrm{HCS}}}=0 .
$$

Since $f_{\text {HCS }}$ has the scaling form (29), it is

$$
\frac{\partial f_{\mathrm{HCS}}}{\partial T_{\mathrm{HCS}}}=-\frac{f_{\mathrm{HCS}}}{2 T_{\mathrm{HCS}}}\left(d+\boldsymbol{V} \cdot \frac{\partial \ln f_{\mathrm{HCS}}}{\partial \boldsymbol{V}}\right),
$$

and, taking into account that $\zeta_{\mathrm{HCS}} \propto T_{\mathrm{HCS}}^{1 / 2}$, Eq. (47) becomes

$$
\begin{gathered}
\frac{\zeta_{\mathrm{HCS}}}{2} \frac{\partial}{\partial \boldsymbol{V}} \cdot\left(\boldsymbol{V} f_{\mathrm{HCS}}\right)-\frac{\zeta_{\mathrm{HCS}}}{2} \frac{\partial}{\partial \boldsymbol{V}} \cdot\left[\boldsymbol{V} f_{\mathrm{HCS}}\left(d+\boldsymbol{V} \cdot \frac{\partial \ln f_{\mathrm{HCS}}}{\partial \boldsymbol{V}}\right)\right] \\
-L\left[f_{\mathrm{HCS}}\left(d+\boldsymbol{V} \cdot \frac{\partial \ln f_{\mathrm{HCS}}}{\partial \boldsymbol{V}}\right)\right]=0
\end{gathered}
$$

Setting $\boldsymbol{u}_{\mathrm{HCS}}=\mathbf{0}$ and transforming to dimensionless variables, the above equation yields

$$
\mathcal{L}^{*}\left(d+\boldsymbol{v}^{*} \cdot \frac{\partial \ln \phi}{\partial \boldsymbol{v}^{*}}\right)=\frac{\zeta_{0}^{*}}{2}\left(d+\boldsymbol{v}^{*} \cdot \frac{\partial \ln \phi}{\partial \boldsymbol{v}^{*}}\right) .
$$

Finally, differentiating Eq. (31) with respect to $\boldsymbol{u}_{\mathrm{HCS}}$, afterwards setting $\boldsymbol{u}_{\mathrm{HCS}}=\mathbf{0}$, and introducing dimensionless variables leads in a similar way to 


$$
\mathcal{L}^{*}\left(\frac{\partial \ln \phi}{\partial \boldsymbol{v}^{*}}\right)=-\frac{\zeta_{0}^{*}}{2}\left(\frac{\partial \ln \phi}{\partial \boldsymbol{v}^{*}}\right) .
$$

The $d+2$ equations (46), (50), and (51), provide exact properties of $\mathcal{L}^{*}$. In fact, Eqs. (50) and (51) are of the form of the eigenvalue problem to be solved at $k=0$, with eigenvalues given by $\zeta_{0}^{*} / 2$ and $-\zeta_{0}^{*} / 2$, respectively. It is straightforward to construct linear combinations of Eqs. (46) and (50) to obtain an additional eigenvalue and eigenvector. The results can then be expressed as [12]

$$
\mathcal{L}^{*} \Psi_{i}(0)=\lambda_{i}(0) \Psi_{i}(0), \quad i=1, \ldots, d+2,
$$

with

$$
\begin{gathered}
\left\{\lambda_{i}(0)\right\}=\left\{0, \frac{\zeta_{0}^{*}}{2},-\frac{\zeta_{0}^{*}}{2}\right\}, \\
\left\{\Psi_{i}(0)\right\}=\left\{d+1+\boldsymbol{v}^{*} \cdot \frac{\partial \ln \phi}{\partial \boldsymbol{v}^{*}},-d-\boldsymbol{v}^{*} \cdot \frac{\partial \ln \phi}{\partial \boldsymbol{v}^{*}},-\hat{\boldsymbol{k}} \cdot \frac{\partial \ln \phi}{\partial \boldsymbol{v}^{*}},\right. \\
\left.-\frac{\partial \ln \phi}{\partial \boldsymbol{v}_{\perp}^{*}}\right\} .
\end{gathered}
$$

The eigenvalue $-\zeta_{0}^{*} / 2$ is $d$-fold degenerated. For convenience for the perturbation calculation to be carried out in the following section, the subspace associated to it has been rearranged. The velocity $\boldsymbol{v}^{*}$ has been decomposed into its component in the direction of $\boldsymbol{k}, v_{\|}^{*}=\hat{\boldsymbol{k}} \cdot \boldsymbol{v}^{*}$, and the remaining $d-1$ ones, forming with it a set of $d$ pairwise orthogonal components, i.e., $\boldsymbol{v}_{\perp}^{*}$ is defined by the $d-1$ components $\boldsymbol{v}^{*} \cdot \hat{\boldsymbol{e}}^{(i)}$. When appropriate, the set of the $d$ eigenfunctions associated with the eigenvalue $-\zeta_{0}^{*} / 2$ will be denoted by $\boldsymbol{\Psi}_{3}(0)$.

Clearly, the above are the long wavelength limit of the hydrodynamic modes defined by Eq. (27). This is a the first primary result of the analysis developed here, demonstration of the existence of hydrodynamic excitations in the spectrum of the linearized Boltzmann equation. The results are exact and apply for arbitrary degree of dissipation.

These long wavelength eigenfunctions are determined from the HCS distribution which depends only on the magnitude of $v^{*}$, so the terms on the right side of Eq. (54) are all determined from $\partial \ln \phi\left(v^{*}\right) / \partial v^{*}$. In the elastic limit, $\phi\left(v^{*}\right)$ becomes Gaussian, and the hydrodynamic eigenfunctions become linear combinations of $1, \boldsymbol{v}^{*}$, and $v^{* 2}$, i.e., of the summational invariants for the conservation laws of mass, momentum, and energy, as expected. For inelastic collisions, the eigenfunctions are quite different, particularly at large $v^{*}$ due to an overpopulation in the HCS distribution relative to the Gaussian. This is illustrated in Figs. 1 and 2, where $\partial \ln \phi(v) / \partial v^{*}$ has been obtained from direct simulation Monte Carlo solution of the Boltzmann equation for the HCS [17].

The set of eigenfunctions $\Psi_{i}(0)$ span a $(d+2)$ dimensional subspace of the Hilbert space, but they are not orthogonal. Consistently, it is easily verified that the operator $\mathcal{L}^{*}$ is not Hermitian. Therefore, it is useful to introduce a biorthogonal set of functions $\Phi_{i}, i=1, \ldots, d+2$, with the requirement

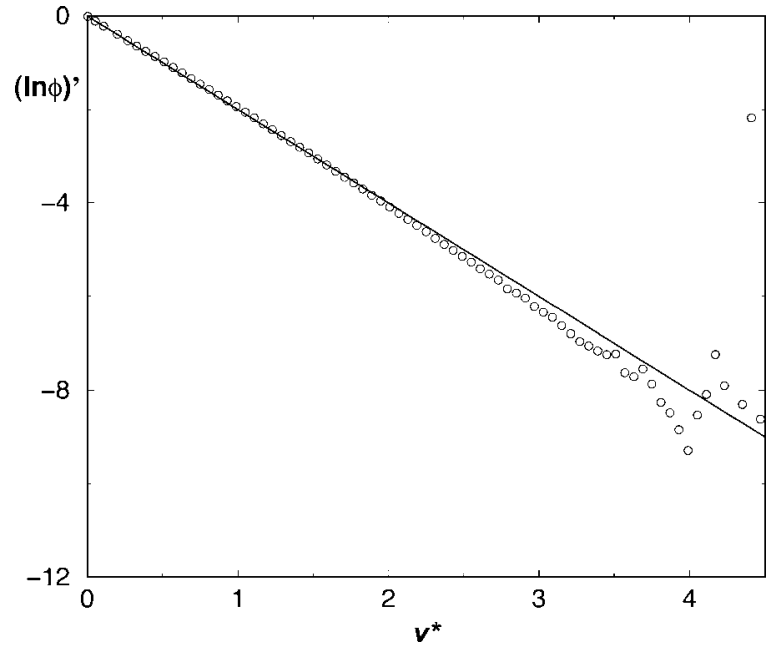

FIG. 1. Plot of $(\ln \phi)^{\prime} \equiv \partial \ln \phi / \partial v^{*}$ as a function of $v^{*}$ for $d$ $=3$ and $\alpha=0.95$. The circles are the numerical derivative of the DSMC results and the solid line is the Gaussian. Quantities are measured in the dimensionless units defined in the text.

$$
\left(\Phi_{i}, \Psi_{j}(0)\right)=\delta_{i j}
$$

To identify the appropriate set of functions, first note that

$$
\left(1, \mathcal{L}^{*} X\right)=0, \quad\left(\boldsymbol{v}^{*}, \mathcal{L}^{*} X\right)=-\frac{\zeta_{0}^{*}}{2}\left(\boldsymbol{v}^{*}, X\right),
$$

as a consequence of the number of particles and momentum conservation in the moment equations (5). Therefore, 1 and $\boldsymbol{v}^{*}$ are eigenfunctions of the adjoint of $\mathcal{L}^{*}, \mathcal{L}^{* \dagger}$, with eigenvalues 0 and $-\zeta_{0}^{*} / 2$, respectively. This gives the set

$$
\left\{\Phi_{i}\right\}=\left\{1, \Phi_{2}, \hat{\boldsymbol{k}} \cdot \boldsymbol{v}^{*}, \boldsymbol{v}_{\perp}^{*}\right\} .
$$

The final choice of $\Phi_{2}$ does not appear to be unique. This is discussed further in Appendix B. For the purposes of the next section, it suffices to make the choice

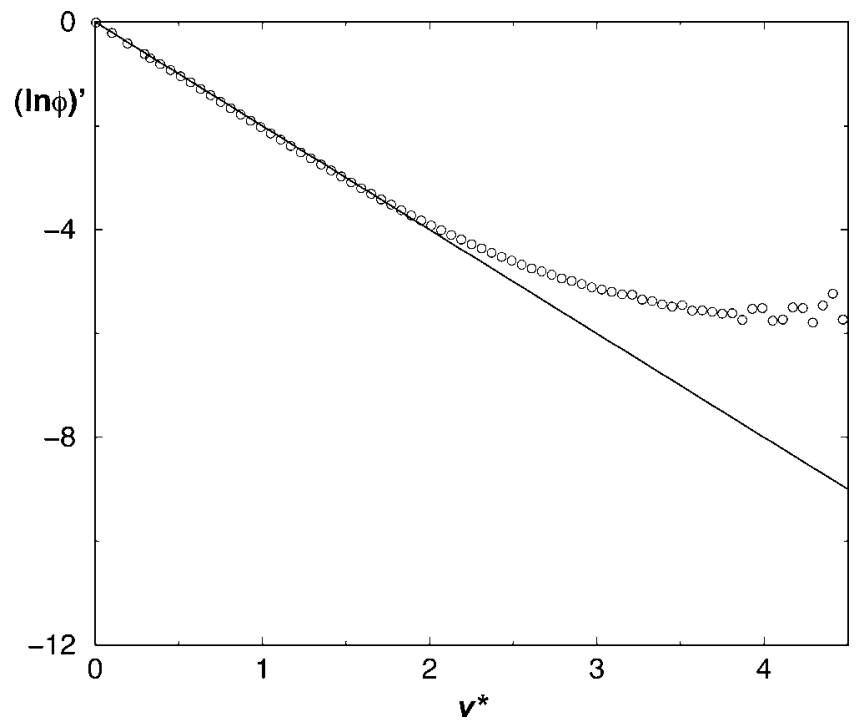

FIG. 2. The same as Fig. 1 but for $\alpha=0.6$. 


$$
\left\{\Phi_{i}\right\}=\left\{1, \frac{v^{* 2}}{d}+\frac{1}{2}, \hat{\boldsymbol{k}} \cdot \boldsymbol{v}^{*}, \boldsymbol{v}_{\perp}^{*}\right\} .
$$

The function $\Phi_{2}$ is not an eigenfunction of the adjoint operator $\mathcal{L}^{* \dagger}$, but it is easily verified that the biorthogonality conditions (55) are satisfied.

\section{NAVIER-STOKES ORDER MODES}

In the previous section, the hydrodynamic modes were identified in the long wavelength limit. Assuming analyticity, their existence at finite wavevectors can be inferred. Furthermore, their explicit construction is possible by perturbation theory. This construction provides a critical test of the internal consistency of other quite different approaches (e.g., the Chapman-Enskog method discussed above and in Appendix A). In particular, the detailed form of the eigenvalues and the dependence of the associated transport coefficients on the restitution coefficient should be exactly the same. This is demonstrated to Navier-Stokes order in this section.

Return now to the eigenvalue problem (43) and consider the case of $k \ll 1$. Look for solutions with the expansion (a more complete characterization of the conditions for this perturbation expansion is given below),

$$
\begin{gathered}
\Psi_{i}(\boldsymbol{k})=\Psi_{i}(0)+k \Psi_{i}^{(1)}+k^{2} \Psi_{i}^{(2)}+\cdots, \\
\lambda_{i}(\boldsymbol{k})=\lambda_{i}(0)+k \lambda_{i}^{(1)}+k^{2} \lambda_{i}^{(2)}+\cdots .
\end{gathered}
$$

The reference eigenfunctions $\Psi_{i}(0)$ and eigenvalues $\lambda_{i}(0)$ are taken to be the long wavelength hydrodynamic results of Eqs. (53) and (54). As already indicated, there is a $d$-fold degeneracy for the eigenvalue $\lambda(0)=-\zeta_{0}^{*} / 2$. However, the $d$-dimensional subspace spanned by its eigenvectors was naturally partitioned by symmetry into the longitudinal and transverse components. The eigenvectors for the transverse modes decouple from the remaining three modes even at finite wave vector for the same symmetry reasons, so there are no complications of degenerate perturbation theory. In the longitudinal subspace, all the eigenvalues are distinct, except in the elastic limit $\alpha=1$, where there is a threefold degeneracy in this subspace. Thus the two cases of unperturbed reference states with $\alpha=1$ and $\alpha<1$ must be distinguished. In the former case, the eigenvalues behave as

$$
\begin{aligned}
\lambda_{i}(\boldsymbol{k}, \alpha)= & {\left[\lambda_{i}(0)+k \lambda_{i}^{(1)}+k^{2} \lambda_{i}^{(2)}+\cdots\right]_{\alpha=1} } \\
& + \text { terms of order }(1-\alpha),
\end{aligned}
$$

and are regular in $(1-\alpha)$. This occurs when the degree of dissipation because of inelasticity is small relative to the effects of the spatial variation. The corresponding eigenvalues are then similar to those of a normal gas, with $d-1$ shear diffusion modes, two sound modes, and a heat diffusion mode. Here attention is restricted to the more interesting and relevant second case of fixed $\alpha<1$ with small spatial perturbations. It will be seen that the modes are now qualitatively different, consistently with the results reported in Sec. II, since the degeneracy of the elastic limit is lifted at the outset by the finite dissipation.

To set up the perturbation expansion, projection operators $\mathcal{P}_{i}$ for the biorthogonal set $\left\{\Phi_{i}, \Psi_{i}(0)\right\}$ are defined by

$$
\mathcal{P}_{i} X=\Psi_{i}(0)\left(\Phi_{i}, X\right),
$$

for an arbitrary element $X$ in the Hilbert space. The eigenvalue problem (43) can be rearranged as

$$
\left[\mathcal{L}^{*}-\lambda_{i}(0)\right] \Psi_{i}(\boldsymbol{k})=\left[\lambda_{i}(k)-\lambda_{i}(0)-i \boldsymbol{k} \cdot \boldsymbol{v}^{*}\right] \Psi_{i}(\boldsymbol{k}) .
$$

Then, operating on both sides of this equation with $\mathcal{Q}_{i}$ $\equiv 1-\mathcal{P}_{i}$, and using the property $\left[\mathcal{L}^{*}-\lambda_{i}(0)\right] \mathcal{P}_{i}=0$, this becomes

$$
\mathcal{Q}_{i}\left[\mathcal{L}^{*}-\lambda_{i}(0)\right] \mathcal{Q}_{i} \Psi_{i}(\boldsymbol{k})=\mathcal{Q}_{i}\left[\lambda_{i}(k)-\lambda_{i}(0)-i \boldsymbol{k} \cdot \boldsymbol{v}^{*}\right] \Psi_{i}(\boldsymbol{k}) .
$$

By construction, the right side of the above equation is orthogonal to the null space for the adjoint of $\left[\mathcal{L}^{*}-\lambda_{i}(0)\right]$, and the Fredholm alternative assures solutions to this equation [18]. The eigenvalue problem for $\Psi_{i}(\boldsymbol{k})$ is determined only up to an overall scale factor, amounting to the choice of normalization. It is convenient to choose

$$
\left(\Phi_{i}, \Psi_{i}(\boldsymbol{k})\right)=1
$$

implying $\left(\Phi_{i}, \Psi_{i}^{(n)}\right)=0$ for $n \geqslant 1$. With this, Eq. (64) gives two sets of equations for the eigenvalues and eigenvectors

$$
\lambda_{i}(\boldsymbol{k})=\lambda_{i}(0)+\left(\Phi_{i}, i \boldsymbol{k} \cdot \boldsymbol{v}^{*} \Psi_{i}(\boldsymbol{k})\right)+\left(\Phi_{i},\left[\mathcal{L}^{*}-\lambda_{i}(0)\right] \mathcal{Q}_{i} \Psi_{i}(\boldsymbol{k})\right)
$$

$$
\begin{aligned}
\mathcal{Q}_{i} \Psi_{i}(\boldsymbol{k})= & \left\{\mathcal{Q}_{i}\left[\mathcal{L}^{*}-\lambda_{i}(\mathbf{0})\right] \mathcal{Q}_{i}\right\}^{-1} \mathcal{Q}_{i}\left[\lambda_{i}(k)-\lambda_{i}(0)\right. \\
& \left.-i \boldsymbol{k} \cdot \boldsymbol{v}^{*}\right] \Psi_{i}(\boldsymbol{k}) .
\end{aligned}
$$

To zeroth order in $k$, these equations give the hydrodynamic modes of the last section in the long wavelength limit, consistently. To first order in $k$, the eigenvectors are

$$
\begin{aligned}
\Psi_{i}^{(1)}(\boldsymbol{k})= & \mathcal{Q}_{i} \Psi_{i}^{(1)}(\boldsymbol{k})=\left\{\mathcal{Q}_{i}\left[\mathcal{L}^{*}-\lambda_{i}(0)\right]\right\}^{-1} \mathcal{Q}_{i}\left(\lambda_{i}^{(1)}\right. \\
& \left.-i \hat{\boldsymbol{k}} \cdot \boldsymbol{v}^{*}\right) \Psi_{i}(0)=-\left\{\mathcal { Q } _ { i } \left[\mathcal{L}^{*}\right.\right. \\
& \left.\left.-\lambda_{i}(0)\right]\right\}^{-1} \mathcal{Q}_{i} i \hat{\boldsymbol{k}} \cdot \boldsymbol{v}^{*} \Psi_{i}(0)
\end{aligned}
$$

The first equality in the above transformations is a consequence of the normalization condition (65). For the first order eigenvalues it is found

$$
\lambda_{i}^{(1)}=\left(\Phi_{i}, i \hat{\boldsymbol{k}} \cdot \boldsymbol{v}^{*} \Psi_{i}(0)\right)+\left(\Phi_{i}, \mathcal{L}^{*} \mathcal{Q}_{i} \Psi_{i}^{(1)}\right)=0
$$

Now the last equality follows from the fact that each $\Phi_{i}$ and $\Psi_{i}(0)$ have the same definite parity under the change $\boldsymbol{v}^{*} \rightarrow-\boldsymbol{v}^{*}$ and the distribution function of the $\operatorname{HCS}, \phi\left(v^{*}\right)$, defining the scalar product is invariant under this change. Thus the first term on the right side vanishes. The second term also vanishes for similar reasons, since $\Phi_{i}$ and $\Psi_{i}^{(1)}$ have opposite parity and $\mathcal{L}^{*}$ is invariant under the change in sign of the velocity.

To second order in $k$ the eigenvectors and eigenvalues are given by

$$
\mathcal{Q}_{i} \Psi_{i}^{(2)}=-\left\{\mathcal{Q}_{i}\left[\mathcal{L}^{*}-\lambda_{i}(0)\right]\right\}^{-1} \mathcal{Q}_{i} i \hat{\boldsymbol{k}} \cdot \boldsymbol{v}^{*} \Psi_{i}^{(1)},
$$




$$
\lambda_{i}^{(2)}=\left(\Phi_{i}, i \boldsymbol{k} \cdot \boldsymbol{v}^{*} \Psi_{i}^{(1)}\right)+\left(\Phi_{i}, \mathcal{L}^{*} \mathcal{Q}_{i} \Psi_{i}^{(2)}\right) .
$$

The above expressions for the eigenvalues are evaluated in Appendix C. The results have the same forms as given in Eqs. (27). Furthermore, the reduced transport coefficients $\eta^{*}, \kappa^{*}$, and $\zeta_{1}^{*}$ are determined from the same integral equations as following from the Chapman-Enskog solution summarized in Appendix A. This confirms that the hydrodynamic modes determined from the spectrum of the linearized Boltzmann equation are consistently determined to NavierStokes order by both methods.

\section{VI. "AGEING" TO HYDRODYNAMICS}

The existence of hydrodynamic excitations only assures that there is a hydrodynamic contribution to the dynamics of small perturbations of the HCS. To establish a description in terms of these hydrodynamic excitations alone, it is necessary to characterize the rest of the excitations in the spectrum. For gases with elastic collisions, it has been shown that for sufficiently small $k$ the hydrodynamic excitations are smaller in magnitude than all other excitations, and bounded away from them $[10,11]$. Consequently, there is a time scale beyond which only the hydrodynamic excitations persist. It is on this space and time scales that hydrodynamics in the usual sense applies. Typically, the conditions are wavelengths larger than the mean free path and times later than the mean free time. This leaves a large domain of macroscopic space and time scales for hydrodynamics.

The extension of this concept of "ageing to hydrodynamics" for granular gases is expected, but its verification is not so straightforward. The mathematical analysis for elastic collisions does not transfer to the granular gas due to the significant differences in the linear collision operator. There are qualitative differences in the hydrodynamic modes. For example, the fact that energy is not conserved means that the hydrodynamic excitations cannot be made arbitrarily small simply by making $k$ small. The fastest decaying hydrodynamic modes is that with eigenvalue $\zeta_{0}^{*} / 2$ at long wavelengths. This has its maximum value at large dissipation, and the question arises as to whether the time scale for this mode can become comparable to or exceed those of the nonhydrodynamic modes at strong dissipation.

\section{A. Model kinetic equation}

Current analysis of the spectrum of the linearized Boltzmann operator for granular gases appears to be limited to the hydrodynamic excitations discussed here, with no characterization of the rest of the spectrum as yet. Consequently, in the remainder of this presentation these questions are addressed in the context of a model kinetic equation. This model [19] is an extension of the familiar Bhatnager, Gross, Krook (BGK) single relaxation time model for normal gases [20]. The Boltzmann equation can be formally written in the form

$$
\left(\frac{\partial}{\partial t}+\boldsymbol{v} \cdot \boldsymbol{\nabla}\right) f=-\nu(f-g) .
$$

There are two significant differences of the model considered here with respect to the original Boltzmann equation. First, the collision frequency is replaced by a velocity independent function of the local density and temperature, $\nu=\nu(n, T)$. Second, the gain term of the Boltzmann equation is replaced by $\nu g$, where $g$ is taken to be a Gaussian function of the velocity

$$
g(\boldsymbol{r}, \boldsymbol{v}, t)=n\left[\frac{b(T)}{\pi}\right]^{d / 2} e^{-b(T) V^{2}} .
$$

Here $\boldsymbol{V}$ is the peculiar velocity defined below Eq. (10). The parameter $b$ is chosen to be function of the local density and temperature so as to enforce the moment conditions (5) above. This leads to the identification

$$
b(T)=\frac{m}{2 T(1-\zeta / \nu)} .
$$

In this way, it is assured that the exact macroscopic balance equations (6)-(10) are preserved by the model. Then the Chapman-Enskog method leads to the same Navier-Stokes hydrodynamic equations as for the Boltzmann equation, with only the transport coefficients being different. In the following, it will be considered that the expression for the collision frequency $\nu$ is chosen as scaling with $n T^{1 / 2}$, in order to mimic the hard sphere behavior. Dimensional analysis then implies the same scaling for the cooling rate $\zeta$. Moreover, note that consistency of the model kinetic equation requires that $\zeta<\nu$ for all values of $\alpha$. A possible choice for the cooling rate is to be the same as obtained from the Boltzmann equation by using a local Maxwellian for the distribution function. In the same spirit, the collision frequency can be fixed by fitting one of the transport coefficients of the model to that obtained from the Boltzamnn equation by the Chapman-Enskog procedure in the first Sonine approximation. If the shear viscosity $\eta$ is used, the above choices lead to $[21]$

$$
\begin{gathered}
\nu(\boldsymbol{r}, t)=\frac{(3-3 \alpha+2 d)(1+\alpha)}{4 d} \nu_{0}(\boldsymbol{r}, t), \\
\zeta(\boldsymbol{r}, t)=\frac{(2+d)\left(1-\alpha^{2}\right)}{4 d} \nu_{0}(\boldsymbol{r}, t),
\end{gathered}
$$

where $\nu_{0}(\boldsymbol{r}, t)$ is an average local collision frequency

$$
\nu_{0}(\boldsymbol{r}, t)=\frac{8 \pi^{(d-1) / 2} n \sigma^{d-1}}{(2+d) \Gamma(d / 2)}\left(\frac{T}{m}\right)^{1 / 2} .
$$

The above expressions yield

$$
\frac{\zeta(\boldsymbol{r}, t)}{\nu(\boldsymbol{r}, t)}=\frac{(2+d)(1-\alpha)}{3-3 \alpha+2 d} \leqslant \frac{2+d}{3+2 d}<1,
$$

in agreement with the model consistency requirement.

This kinetic model reduces to the BGK model for normal gases at $\alpha=1$ [20]. Otherwise it reproduces all of the qualitative features of the granular Boltzmann equation, including a nontrivial HCS and the same hydrodynamic excitations discussed in the sections above. In some respects, the model kinetic equation is more complex than the Boltzmann equation since the collision operator is a nonlinear functional of $f$ through the dependence of $g$ on $T$ and $\boldsymbol{u}$. However, the lin- 
earized model kinetic equation for small perturbations of the HCS is considerably simpler than that for the Boltzmann equation, as shown below.

\section{B. Model HCS and linear model kinetic equation}

The HCS equation (31) for this model becomes in dimensionless form

$\frac{\zeta_{0}^{*}}{2} \frac{\partial}{\partial \boldsymbol{v}^{*}} \cdot\left(\boldsymbol{v}^{*} \phi\right)+\nu_{0}^{*} \phi=\nu_{0}^{*}\left[\pi\left(1-\zeta_{0}^{*} / \nu_{0}^{*}\right)\right]^{-d / 2} \exp \left(-\frac{v^{* 2}}{1-\zeta_{0}^{*} / \nu_{0}^{*}}\right)$,

where $\zeta_{0}^{*} \equiv \ell \zeta_{\mathrm{HCS}} / v_{\mathrm{HCS}}(t)$, as in the preceding sections and, consistently, $\nu_{0}^{*} \equiv \ell \nu_{\mathrm{HCS}} / v_{\mathrm{HCS}}(t)$. The solution to the above equation is

$$
\begin{aligned}
\phi\left(v^{*}\right)= & \nu_{0}^{*}\left[\pi\left(1-\zeta_{0}^{*} / \nu_{0}^{*}\right)\right]^{-d / 2} \int_{0}^{\infty} d s \\
& \times \exp \left[-\left(\frac{d \zeta_{0}^{*}}{2}+\nu_{0}^{*}\right) s\right] \exp \left(-\frac{e^{-\zeta_{0}^{*} v^{* 2}}}{1-\zeta_{0}^{*} / \nu_{0}^{*}}\right) .
\end{aligned}
$$

It is easily verified that this distribution exhibits algebraic decay for large velocities

$$
\phi\left(v^{*}\right) \sim \frac{p \pi^{-d / 2}}{2}\left(\frac{p}{p-2}\right)^{-p / 2} \Gamma\left(\frac{p+d}{2}\right) v^{*-(p+d)},
$$

with $p=2 \nu_{0}^{*} / \zeta_{0}^{*}$. Therefore, moments of degree $p$ or greater do not exist.

The linearized kinetic model equation for small perturbations of the HCS is obtained in Appendix D, with the result, in the dimensionless variables of the previous sections,

$$
\left(\partial_{s}+i \boldsymbol{k} \cdot \boldsymbol{v}^{*}+\mathcal{L}_{m}^{*}\right) \tilde{\Delta}\left(\boldsymbol{k}, \boldsymbol{v}^{*}, s\right)=0 .
$$

The linear collision operator in this case is

$$
\mathcal{L}_{m}^{*}=\sum_{i} \lambda_{i}(0) \mathcal{P}_{i}+\mathcal{Q}\left(\nu_{\mathrm{HCS}}^{*}+\frac{\zeta_{0}^{*}}{2} \phi^{-1} \frac{\partial}{\partial \boldsymbol{v}^{*}} \cdot \boldsymbol{v}^{*} \phi\right) \mathcal{Q}
$$

The projection operators $\mathcal{P}_{i}$ are the same as defined in Eq. (62) and

$$
\mathcal{P} \equiv \sum_{i} \mathcal{P}_{i}, \quad \mathcal{Q} \equiv 1-\mathcal{P} .
$$

In the above expressions, the summations are over the hydrodynamic modes. The first term on the right-hand side of Eq. (83) is the projection onto the hydrodynamic eigenfunctions, while the second one is orthogonal to this subspace. Consequently, the spectrum of $\mathcal{L}_{m}^{*}$ for the model kinetic equation has the same $k=0$ hydrodynamic eigenfunctions and eigenvalues as the Boltzmann equation, i.e., it is

$$
\mathcal{L}_{m}^{*} \Psi_{i}(0)=\lambda_{i}(0) \Psi_{i}(0), \quad i=1, \ldots, d+2 .
$$

Furthermore, the structure of $\mathcal{L}_{m}^{*}$ decomposes into operators defined in the hydrodynamic subspace and its orthogonal complement. This allows more detailed analysis of the nonhydrodynamic spectrum. The associated eigenfunctions lie in the orthogonal complement $\mathcal{Q}$. Consider the general form for $\Psi_{Q}=\mathcal{Q} \Psi$

$$
\begin{aligned}
\left(\Psi_{Q},\left[\mathcal{L}^{*}-\sum_{i} \lambda_{i}(0) \mathcal{P}_{i}\right] \Psi_{Q}\right) \\
=\left(\Psi_{Q},\left(\nu_{0}^{*}+\frac{\zeta_{0}^{*}}{2} \phi^{-1} \frac{\partial}{\partial \boldsymbol{v}^{*}} \cdot \boldsymbol{v}^{*} \phi\right) \Psi_{Q}\right) \\
=\left(\left(\nu_{0}^{*}-\frac{\zeta_{0}^{*}}{2} \boldsymbol{v}^{*} \cdot \frac{\partial}{\partial \boldsymbol{v}^{*}}\right) \Psi_{Q}, \Psi_{Q}\right) .
\end{aligned}
$$

The second term in the scalar product above can be simplified as

$$
\begin{aligned}
\frac{\zeta_{0}^{*}}{2}\left(\boldsymbol{v}^{*} \cdot \frac{\partial}{\partial \boldsymbol{v}^{*}} \Psi_{Q}, \Psi_{Q}\right) & =\frac{\zeta_{0}^{*}}{4}\left(1, \boldsymbol{v}^{*} \cdot \frac{\partial}{\partial \boldsymbol{v}^{*}} \Psi_{Q}^{2}\right) \\
& =-\frac{\zeta_{0}^{*}}{4}\left(\phi^{-1} \frac{\partial}{\partial \boldsymbol{v}^{*}} \cdot\left(\boldsymbol{v}^{*} \phi\right), \Psi_{Q}^{2}\right) \\
& =\frac{\nu_{0}^{*}}{2}\left(\Psi_{Q}, \Psi_{Q}\right)-\frac{\nu_{0}^{*}}{2}\left(\phi^{-1} g_{0}^{*}, \Psi_{Q}^{2}\right),
\end{aligned}
$$

where

$$
g_{0}^{*}\left(v^{*}\right)=n_{\mathrm{HCS}}^{-1} v^{d}(t) g_{\mathrm{HCS}}(v, t) \geqslant 0 .
$$

Then Eq. (86) gives the desired inequality

$$
\begin{aligned}
\frac{\left(\Psi_{Q},\left(\mathcal{L}_{m}^{*}-\sum_{i} \lambda_{i}(0) \mathcal{P}_{i}\right] \Psi_{Q}\right)}{\left(\Psi_{Q}, \Psi_{Q}\right)} & =\frac{\nu_{0}^{*}}{2}\left[1+\frac{\left(\Psi_{Q}, \phi^{-1} g_{0}^{*} \Psi_{Q}\right)}{\left(\Psi_{Q}, \Psi_{Q}\right)}\right] \\
& >\frac{\nu_{0}^{*}}{2} .
\end{aligned}
$$

Therefore, the nonhydrodynamic spectrum of $\mathcal{L}_{m}^{*}$ consists of points (or continuum) with real parts larger than $\nu_{0}^{*} / 2$. The fastest decaying hydrodynamic eigenvalue is at $\zeta_{0}^{\prime} / 2$. The hydrodynamic excitations are isolated from the rest of the spectrum for $\zeta_{0}^{*}<\nu_{0}^{*}$, as is always the case. Assuming analyticity in $k$, the hydrodynamic modes at finite wavelength also will be isolated from the rest of the spectrum for sufficiently small $k$.

In summary, the kinetic model considered here illustrates the expected behavior for the Boltzmann equation. The linearized kinetic equation for small perturbations of the HCS characterizes the complete complex response. Among the excitations there are $d+2$ hydrodynamic modes. At long wavelengths, these modes are isolated from and have smaller eigenvalues than the rest of the spectrum. Hence, there is a sufficiently long time scale on which only the hydrodynamic excitations persist.

This kinetic model can be used to explore the role of hydrodynamics in great detail as the linear equation can be solved exactly [21]. For example, it has been shown that the hydrodynamic modes extend to very short wavelengths far beyond the validity of the Navier-Stokes approximation. 


\section{DISCUSSION}

The objective here has been to explore the role of hydrodynamics for a granular gas by a direct analysis of the spectrum of the linear inelastic Boltzmann equation. This analysis has been shown to lead to results equivalent to those obtained previously based on the Chapman-Enskog method to solve the Boltzmann equation. However, the current method is more straightforward and less susceptible to subjective questions about the applicability of the ChapmanEnskog method to granular gases. In addition, the formulation of the problem in terms of the spectrum of the linearized collision operator is the proper setting to explore the context for dominance of a hydrodynamic description.

The eigenvalue problem posed in Sec. IV, together with the macroscopic balance equations leads to a precise definition of the hydrodynamic modes. Here, it has been established that they exist for sufficiently long wavelengths. It remains to explore the form and extent to which they are meaningful at shorter wavelengths, and other methods to solve the eigenvalue problem are available to complement the simple perturbation theory applied here. This has been done in Ref. [21] for the kinetic model introduced in Sec. V, demonstrating the extension of the hydrodynamic modes to wavelengths an order of magnitude shorter than those required for the Navier-Stokes approximation .

A second relevant result of the analysis here is the identification of the hydrodynamic eigenfunctions. This allows calculation of the hydrodynamic component for properties of interest, and provides the means to explore dynamical mechanisms beyond the Boltzmann description based on hydrodynamics (e.g., mode coupling phenomena for fluctuations). For normal gases, these eigenfunctions are the summational invariants $\left(1, v^{2}, \boldsymbol{v}\right)$ in the long wavelength limit. For a granular gas, they are replaced by derivatives of the logarithm of the HCS distribution function, which behave quite differently for large velocities, as illustrated in Figs. 1 and 2 .

The hydrodynamic excitations are usually interpreted as a set of $d+2$ modes associated with the density, temperature, and flow field of the macroscopic balance equations. Here, they are simply $d+2$ points associated with the spectrum of the linearized inelastic Boltzmann equation for response to small perturbations. It is often claimed that the temperature should not be included in the set of hydrodynamic fields, as the energy is not conserved. However, the analysis here shows that in the proper reduced variables all but one eigenvalue is nonzero in the long wavelength limit. Thus, the exclusion of one field from the description will not recover a smaller set of eigenvalues clustered around zero at long wavelengths. Instead, the $d+2$ modes have the following qualitative behavior. They are all clustered near zero for weak dissipation, but become increasingly separated at stronger dissipation. This means that within the hydrodynamic description there are time scales set by both the wavelength and the cooling rate and these can be quite different.

A second question is the isolation of these hydrodynamic modes from the rest of the spectrum. At weak dissipation, it is expected that this is the case since the hydrodynamic eigenvalues are all small and the nonhydrodynamic spectrum is expected to be of the order of the collision frequency. At strong dissipation, there is a hydrodynamic mode of the order of $\zeta_{0}^{*} / 2$, which can be of the order of the collision frequency. It remains an open question regarding the size of this eigenvalue relative to the nonhydrodynamic spectrum. It is possible that the isolation of the hydrodynamic spectrum places some restriction on the degree of dissipation. However, the analysis based on the kinetic model of Sec. VI suggests this may not be the case. For the kinetic model, all of the hydrodynamic spectra remain isolated from all of the nonhydrodynamic spectra for any degree of dissipation. In this case, the $d+2$ hydrodynamic modes define a dominant set on a sufficiently large time scale, even when the separation of times among the hydrodynamic modes is significant.

The idealized model for a granular gas discussed here can be made more realistic by considering a more complex binary collision rule. In particular, a velocity dependent restitution coefficient (which approaches unity as the relative velocity goes to zero) and tangential friction are two additional qualitative features of real granular fluids. However, the general form of the hydrodynamic equations (macroscopic balance equations) is unchanged in that case; only the detailed values of the transport coefficients, pressure, and cooling rates differ from those of the present model. Similarly, the collision operator of the Boltzmann equation becomes more complex but its properties relevant for the macroscopic balance equations are the same. Most of the analysis given here depends only on those properties rather than the detailed form of the collision operator. Consequently, it is expected that the implications of our simple model extend to more realistic models as well.

The analysis of the linear Boltzmann collision operator for normal, elastic gases is quite complete [10]. It is hoped that the beginning provided here for granular gases will provoke the new mathematical analysis required for this case as well.

\section{ACKNOWLEDGMENTS}

J.W.D. thanks A. Baskaran for helpful discussions and both authors thank M.J. Ruiz-Montero for providing Figs. 1 and 2. The research of J.W.D. was supported in part by Department of Energy Grant No. DE-FG02ER54677. The research of J.J.B. was supported by the Ministerio de Educación y Ciencia (Spain) through Grant No. BFM200501398 (partially financed by FEDER funds).

\section{APPENDIX A: CHAPMAN-ENSKOG RESULTS}

The Chapman-Enskog method constructs a solution to the Boltzmann equation whose space and time dependence occurs entirely through the hydrodynamic fields and their gradients. For small spatial gradients, i.e., small relative variation of the hydrodynamic fields over a mean free path, the solution reads (we use the same notation as in Ref. [5])

$$
\begin{aligned}
f(\boldsymbol{r}, \boldsymbol{v}, t)= & f_{\mathrm{HCS}}^{(0)}(\boldsymbol{r}, \boldsymbol{v}, t)+\mathcal{A} \cdot \boldsymbol{\nabla} \ln T+\mathcal{B} \cdot \boldsymbol{\nabla} \ln n+\mathrm{C}: \boldsymbol{\nabla} \boldsymbol{u} \\
& +\mathcal{M} \boldsymbol{\nabla}^{2} T+\mathcal{N} \boldsymbol{\nabla}^{2} n .
\end{aligned}
$$

Consider the contributions to the heat flux and the momen- 
tum flux from the terms of first order in the gradients of the above expression. Since these fluxes appear in the macroscopic balance equations under a gradient, the resulting contributions are of second order in the gradients (Navier-Stokes order). For consistency, the cooling rate which does not occur under a gradient in the balance equations must be calculated to second order. Therefore, the last two terms on the right hand side of Eq. (A1) give contributions to the cooling rate at this order, but lead to higher order (Burnett) terms in the heat and momentum fluxes. Additional nonlinear terms of second order in the gradients coming from the cooling rate have been omitted in Eq. (A1) as only the linear hydrodynamic equations are considered here. The reference distribution function $f_{\mathrm{HCS}}^{(0)}(\boldsymbol{r}, \boldsymbol{v}, t)$ has the same functional form as the distribution function of the HCS discussed at the beginning of Sec. III, but scaled with respect to the local exact hydrodynamic fields at time $t$ for the generally nonuniform, nonstationary state. Therefore, it can be considered as the local HCS distribution function.

The dissipative part of the pressure tensor and the heat flux are given by Eqs. (11) and (12), respectively. The transport coefficients in these equations are determined from the functions $\mathcal{A}, \mathcal{B}$, and $\mathrm{C}$ appearing in Eq. (A1)) through

$$
\begin{gathered}
\eta=-\frac{1}{d^{2}+d-2} \int d \boldsymbol{v} \mathrm{D}(\boldsymbol{v}): \mathrm{C}(\boldsymbol{v}), \\
\kappa=-\frac{1}{d T} \int d \boldsymbol{v} S(\boldsymbol{v}) \cdot \mathcal{A}(\boldsymbol{v}), \\
\mu=-\frac{1}{d n} \int d \boldsymbol{v} S(\boldsymbol{v}) \cdot \mathcal{B}(\boldsymbol{v}),
\end{gathered}
$$

where the functions $\mathrm{D}(\boldsymbol{v})$ and $S(\boldsymbol{v})$ are defined by

$$
\mathrm{D} \equiv m\left(\boldsymbol{v} \boldsymbol{v}-\frac{v^{2}}{d} \mathrm{I}\right), \quad \boldsymbol{S}=\left(\frac{m v^{2}}{2}-\frac{d+2}{2} T\right) \boldsymbol{v} .
$$

The functions $\mathcal{A}(\boldsymbol{v}, n, T), \mathcal{B}(\boldsymbol{v}, n, T)$, and $\mathrm{C}(\boldsymbol{v}, n, t)$ are solutions of the integral equations

$$
\begin{gathered}
\left(-\zeta_{\mathrm{HCS}}^{(0)} T \frac{\partial}{\partial T}+L^{(0)}-\frac{\zeta_{\mathrm{HCS}}^{(0)}}{2}\right) \mathcal{A}=\boldsymbol{A}, \\
\left(-\zeta_{\mathrm{HCS}}^{(0)} T \frac{\partial}{\partial T}+L^{(0)}\right) \mathcal{B}=\boldsymbol{B}+\zeta_{\mathrm{HCS}}^{(0)} \mathcal{A}, \\
\left(-\zeta_{\mathrm{HCS}}^{(0)} T \frac{\partial}{\partial T}+L^{(0)}\right) \mathrm{C}=\mathrm{G},
\end{gathered}
$$

with the definitions

$$
\begin{gathered}
L^{(0)}(t) X(\boldsymbol{v})=-J\left[f_{\mathrm{HCS}}^{(0)}, X\right]-J\left[X, f_{\mathrm{HCS}}^{(0)}\right], \\
\boldsymbol{A}=\frac{\boldsymbol{v}}{2} \frac{\partial}{\partial \boldsymbol{v}} \cdot\left(\boldsymbol{v} f_{\mathrm{HCS}}^{(0)}\right)-\frac{T}{m} \frac{\partial f_{\mathrm{HCS}}^{(0)}}{\partial \boldsymbol{v}}, \quad \boldsymbol{B}=-\boldsymbol{v} f_{\mathrm{HCS}}^{(0)}-\frac{T}{m} \frac{\partial f_{\mathrm{HCS}}^{(0)}}{\partial \boldsymbol{v}}, \\
\mathrm{G}=\frac{\partial}{\partial \boldsymbol{v}}\left(\boldsymbol{v} f_{\mathrm{HCS}}^{(0)}\right)-\frac{1}{d} \frac{\partial}{\partial \boldsymbol{v}} \cdot\left(\boldsymbol{v} f_{\mathrm{HCS}}^{(0)}\right) .
\end{gathered}
$$

Moreover, $\zeta_{\mathrm{HCS}}^{(0)}$ is the local form of $\zeta_{\mathrm{HCS}}$.
The transport coefficients arising from the cooling rate are best described by first making the expression for the cooling rate more explicit. In general, it is given by a bilinear functional of the distribution function

$$
\zeta=\zeta[f, f],
$$

where

$$
\begin{aligned}
\zeta[X, Y] & =-\frac{m}{d n T} \int d \boldsymbol{v} v^{2} J[X, Y] \\
& =\left(1-\alpha^{2}\right) \frac{m \pi^{(d-1) / 2} \sigma^{d-1}}{4 d n T \Gamma\left(\frac{d+3}{2}\right)} \int d \boldsymbol{v} \int d \boldsymbol{v}_{1} g^{3} X(\boldsymbol{v}) Y\left(\boldsymbol{v}_{1}\right) \\
& =\xi[Y, X] .
\end{aligned}
$$

The linear contributions from the cooling rate to second order order in the gradients are then

$$
\zeta_{L}^{(2)}=\zeta_{1} \nabla^{2} T+\zeta_{2} \nabla^{2} n,
$$

with

$$
\zeta_{1}=2 \zeta\left[\mathcal{M}, f_{\mathrm{HCS}}^{(0)}\right], \quad \zeta_{2}=2 \zeta\left[\mathcal{N}, f_{\mathrm{HCS}}^{(0)}\right] .
$$

The integral equations for $\mathcal{M}$ and $\mathcal{N}$ are found to be

$$
\begin{aligned}
\left(-\zeta_{\mathrm{HCS}}^{(0)} T \frac{\partial}{\partial T}-\frac{3}{2} \zeta_{\mathrm{HCS}}^{(0)}+L^{(0)}\right) \mathcal{M}=T \zeta_{1} \frac{\partial f_{\mathrm{HCS}}^{(0)}}{\partial T} & -\left(\frac{2 \kappa}{d n} \frac{\partial f_{\mathrm{HCS}}^{(0)}}{\partial T}+\frac{1}{d T} \mathcal{A} \cdot \boldsymbol{v}\right) \\
\left(-\zeta_{\mathrm{HCS}}^{(0)} T \frac{\partial}{\partial T}+L^{(0)}\right) \mathcal{N} & =T \zeta_{2} \frac{\partial f_{\mathrm{HCS}}^{(0)}}{\partial T}+\frac{T \zeta_{\mathrm{HCS}}^{(0)}}{n} \mathcal{M} \\
& -\left(\frac{2 \mu}{d n} \frac{\partial f_{\mathrm{HCS}}^{(0)}}{\partial T}+\frac{1}{d n} \mathcal{B} \cdot \boldsymbol{v}\right)
\end{aligned}
$$

The above results can be easily transformed to be expressed in terms of the reduced units and quantities introduced in Secs. II and III. Dimensionless transport coefficients are again defined by Eqs. (23) and (24), but replacing $n_{\mathrm{HCS}}$ and $T_{\mathrm{HCS}}$ by their local values $n$ and $T$. Of course, now it is $\ell=\left(n \sigma^{d-1}\right)^{-1}$ and $v_{\mathrm{HCS}}(t)$ is replaced by $v(t)=(2 T / m)^{1 / 2}$. In this way, it is obtained

$$
\eta^{*}=\frac{1}{d^{2}+d-2} \sum_{i, j}\left(D_{i j}^{*}, C_{i j}^{*}\right), \quad \kappa^{*}=\frac{1}{d^{2}} \sum_{i}\left(S_{i}^{*}, \mathcal{A}_{i}^{*}\right),
$$

$$
\mu^{*}=\frac{1}{d^{2}} \sum_{i}\left(S_{i}^{*}, \mathcal{B}_{i}^{*}\right),
$$

with the dimensionless functions $\mathrm{D}^{*}$ and $S^{*}$ given by

$$
\mathrm{D}^{*}=\boldsymbol{v}^{*} \boldsymbol{v}^{*}-\frac{v^{* 2}}{d} \mathrm{l}, \quad \boldsymbol{S}^{*}=\left(v^{* 2}-\frac{d+2}{2}\right) \boldsymbol{v}^{*} .
$$

Moreover, $\mathcal{A}^{*}, \mathcal{B}^{*}$, and $\mathrm{C}^{*}$ are now defined through the equations 


$$
\begin{aligned}
& \left(\mathcal{L}^{*}-\frac{\zeta_{0}^{*}}{2}\right) \mathcal{A}^{*}=\boldsymbol{A}^{*}, \\
& \mathcal{L}^{*} \mathcal{B}^{*}=\boldsymbol{B}^{*}+\zeta_{0}^{*} \mathcal{A}^{*}, \\
& \left(\mathcal{L}^{*}+\frac{\zeta_{0}^{*}}{2}\right) \mathcal{C}^{*}=\mathrm{G}^{*},
\end{aligned}
$$

where the operator $\mathcal{L}^{*}$ is defined in Eq. (37) and

$$
\begin{gathered}
\boldsymbol{A}^{*}=\boldsymbol{v}^{*} \Psi_{2}(0)-\boldsymbol{\Psi}_{3}(0), \quad \boldsymbol{B}^{*}=2 \boldsymbol{v}^{*}-\boldsymbol{\Psi}_{3}(0), \\
\mathrm{G}^{*}=\boldsymbol{v}^{*} \boldsymbol{\Psi}_{3}(0)-\frac{\mathrm{I}}{d} \boldsymbol{v}^{*} \cdot \boldsymbol{\Psi}_{3}(0)
\end{gathered}
$$

The expressions of the reduced transport coefficients $\zeta_{1}^{*}$ and $\zeta_{2}^{*}$ are

$$
\zeta_{1}^{*}=\frac{1}{d}\left(a, \mathcal{M}^{*}\right), \quad \zeta_{2}^{*}=\frac{1}{d}\left(a, \mathcal{N}^{*}\right),
$$

where

$$
a\left(\boldsymbol{v}^{*}\right)=\frac{\left(1-\alpha^{2}\right) \pi^{(d-1) / 2}}{2 \Gamma\left(\frac{d+3}{2}\right)} \int d \boldsymbol{v}_{1}^{*} \phi\left(v_{1}^{*}\right) g^{* 3} .
$$

The integral equations obeyed by $\mathcal{M}^{*}$ and $\mathcal{N}^{*}$ are

$$
\begin{aligned}
& \left(\mathcal{L}^{*}-\frac{\zeta_{0}^{*}}{2}\right) \mathcal{M}^{*}=\left(\zeta_{1}^{*}-\kappa^{*}\right) \Psi_{2}(0)+\frac{1}{d} \mathcal{A}^{*} \cdot \boldsymbol{v}^{*}, \\
& \mathcal{L}^{*} \mathcal{N}^{*}=\zeta_{0}^{*} \mathcal{M}^{*}+\left(\zeta_{2}^{*}-\mu^{*}\right) \Psi_{2}(0)+\frac{1}{d} \mathcal{B}^{*} \cdot \boldsymbol{v}^{*} .
\end{aligned}
$$

For later use, it is convenient to elaborate more the above expression for $\zeta_{1}^{*}$. By construction, the velocity integrals of $\mathcal{M}$ times $1, \boldsymbol{v}$, and $v^{2}$ vanish. This is equivalent to say that $\mathcal{M}^{*}$ is orthogonal to the set of functions $\Phi_{i}$ defined in Eq. (58), and in particular it verifies $\mathcal{M}^{*}=\mathcal{Q}_{2} \mathcal{M}^{*}$, with $\mathcal{Q}_{2}=1$ $-\mathcal{P}_{2}, \mathcal{P}_{i}$ being the projection operator defined in Eq. (62). Then, acting with $\mathcal{Q}_{2}$ on both sides of Eq. (A23) it is obtained

$$
\mathcal{M}^{*}=\mathcal{Q}_{2} \mathcal{M}^{*}=\frac{1}{d}\left[\mathcal{Q}_{2}\left(\mathcal{L}^{*}-\frac{\zeta_{0}^{*}}{2}\right)\right]^{-1} \mathcal{Q}_{2} \mathcal{A}^{*} \cdot \boldsymbol{v}^{*}
$$

For the same reason, Eq. (A17) yields

$$
\begin{aligned}
\mathcal{A}^{*}= & \mathcal{Q}_{2} \mathcal{A}^{*} \\
= & {\left[\mathcal{Q}_{2}\left(\mathcal{L}^{*}-\frac{\zeta_{0}}{2}\right)\right]^{-1} \mathcal{Q}_{2} A^{*} } \\
= & {\left[\mathcal{Q}_{2}\left(\mathcal{L}^{*}-\frac{\zeta_{0}^{*}}{2}\right)\right]^{-1} \mathcal{Q}_{2} \boldsymbol{v}^{*} \Psi_{2}(0) } \\
& -\left[\mathcal{Q}_{2}\left(\mathcal{L}^{*}-\frac{\zeta_{0}}{2}\right)\right]^{-1} \boldsymbol{\Psi}_{3}(0)
\end{aligned}
$$

$$
=\left[\mathcal{Q}_{2}\left(\mathcal{L}^{*}-\frac{\zeta_{0}^{*}}{2}\right)\right]^{-1} \mathcal{Q}_{2} \boldsymbol{v}^{*} \Psi_{2}(0)+\frac{1}{\zeta_{0}^{*}} \boldsymbol{\Psi}_{3}(0) .
$$

Substitution of this expression into Eq. (A25) after some algebra leads to

$$
\begin{aligned}
\mathcal{M}^{*}= & \frac{1}{d}\left[\mathcal{Q}_{2}\left(\mathcal{L}^{*}-\frac{\zeta_{0}^{*}}{2}\right)\right]^{-1} \\
& \times \mathcal{Q}_{2} \boldsymbol{v}^{*} \cdot\left[\mathcal{Q}_{2}\left(\mathcal{L}^{*}-\frac{\zeta_{0}^{*}}{2}\right)\right]^{-1} \mathcal{Q}_{2} \boldsymbol{v}^{*} \Psi_{2}(0)-\frac{2}{\zeta_{0}^{* 2}} \Psi_{1}(0),
\end{aligned}
$$

and use of this into Eq. (A21) gives the result

$$
\zeta_{1}^{*}=\frac{1}{\zeta_{0}^{*}}+\frac{1}{d}\left(a, \mathcal{M}_{1}^{*}\right)
$$

with

$$
\begin{aligned}
\mathcal{M}_{1}^{*}= & \frac{1}{d}\left[\mathcal{Q}_{2}\left(\mathcal{L}^{*}-\frac{\zeta_{0}^{*}}{2}\right)\right]^{-1} \\
& \times \mathcal{Q}_{2} \boldsymbol{v}^{*} \cdot\left[\mathcal{Q}_{2}\left(\mathcal{L}^{*}-\frac{\zeta_{0}^{*}}{2}\right)\right]^{-1} \mathcal{Q}_{2} \boldsymbol{v}^{*} \Psi_{2}(0) .
\end{aligned}
$$

Although the expression for $\zeta_{2}^{*}$ can be written in a similar way, it will not be needed here.

\section{APPENDIX B: ADJOINT LINEAR OPERATOR AND BIORTHOGONAL SET}

The adjoint for $\mathcal{L}^{*}, \mathcal{L}^{* \dagger}$, is defined as usual by

$$
\left(X, \mathcal{L}^{*} Y\right)=\left(\mathcal{L}^{* \dagger} X, Y\right)
$$

for arbitrary $X\left(\boldsymbol{v}^{*}\right)$ and $Y\left(\boldsymbol{v}^{*}\right)$ belonging to the Hilbert space. The explicit form of $\mathcal{L}^{*}$ is given in Eq. (37). From it, and using the above definition, the expression for $\mathcal{L}^{* \dagger}$ is easily found,

$$
\begin{aligned}
\mathcal{L}^{* \dagger} X\left(\boldsymbol{v}^{*}\right)= & -\int d \boldsymbol{v}_{1}^{*} \phi\left(v_{1}^{*}\right) \int d \hat{\boldsymbol{\sigma}} \Theta(\hat{\boldsymbol{\sigma}} \cdot \boldsymbol{g}) \hat{\boldsymbol{\sigma}} \cdot \boldsymbol{g}\left[X\left(\boldsymbol{v}^{* \prime \prime}\right)\right. \\
& \left.+X\left(\boldsymbol{v}_{1}^{* \prime \prime}\right)-X\left(\boldsymbol{v}^{*}\right)-X\left(\boldsymbol{v}_{1}^{*}\right)\right]-\frac{\zeta_{0}^{*}}{2} \boldsymbol{v}^{*} \cdot \frac{\partial}{\partial \boldsymbol{v}^{*}} X\left(\boldsymbol{v}^{*}\right),
\end{aligned}
$$

where $\boldsymbol{v}^{* \prime \prime}$ and $\boldsymbol{v}_{1}^{* \prime \prime}$ are the postcollisional velocities corresponding to $\boldsymbol{v}^{*}$ and $\boldsymbol{v}_{1}^{*}$,

$$
\begin{aligned}
& \boldsymbol{v}^{* \prime \prime}=\boldsymbol{v}^{*}-\frac{1+\alpha}{2}\left(\hat{\boldsymbol{\sigma}} \cdot g^{*}\right) \hat{\boldsymbol{\sigma}} \\
& \boldsymbol{v}_{1}^{* \prime \prime}=\boldsymbol{v}_{1}^{*}+\frac{1+\alpha}{2}\left(\hat{\boldsymbol{\sigma}} \cdot g^{*}\right) \hat{\boldsymbol{\sigma}} .
\end{aligned}
$$

Equation (B2) gives immediately 


$$
\mathcal{L}^{* \dagger} 1=0, \quad \mathcal{L}^{* \dagger} \boldsymbol{v}^{*}=-\frac{\zeta_{0}^{*}}{2} \boldsymbol{v}^{*},
$$

so that 1 and $\boldsymbol{v}^{*}$ are eigenfunctions of the adjoint operator with eigenvalues 0 and $-\zeta_{0}^{*} / 2$, respectively.

However, the kinetic energy is not an eigenfunction of the adjoint operator. Direct calculation gives

$$
\mathcal{L}^{* \dagger} v^{* 2}=-\zeta_{0}^{*} v^{* 2}+\frac{a\left(\boldsymbol{v}^{*}\right)}{2},
$$

where $a(\boldsymbol{v})$ is given by Eq. (A22). Nevertheless, a biorthogonal set can be constructed from $1, \boldsymbol{v}^{*}$, and $v^{* 2}$ and it is given in Eq. (58). As noted in the main text, the choice of this set is not unique. The conditions of biorthogonality on $\Phi_{2}$ are

$$
\begin{gathered}
\left(\Phi_{2}, \Psi_{2}(0)\right)=\left(\boldsymbol{v}^{*} \cdot \frac{\partial \Phi_{2}}{\partial \boldsymbol{v}^{*}}, 1\right)=1, \\
\left(\Phi_{2}, \Psi_{1}(0)\right)=\left(\Phi_{2}, 1\right)-1=0, \\
\left(\Phi_{2}, \Psi_{3}(0)\right)=\left(\frac{\partial \Phi_{2}}{\partial \boldsymbol{v}^{*}}, 1\right)=\mathbf{0} .
\end{gathered}
$$

A sufficient condition to guarantee that the above relations are verified, is that $\Phi_{2}$ have the form

$$
\Phi_{2}\left(\boldsymbol{v}^{*}\right)=A+B b\left(v^{*}\right)
$$

where $b\left(v^{*}\right)$ is an arbitrary scalar function of $\boldsymbol{v}^{*}$, and the constants $A$ and $B$ are determined from

$$
A=1-(1, b) B, \quad B=\left(\boldsymbol{v}^{*} \cdot \frac{\partial b}{\partial \boldsymbol{v}^{*}}, 1\right)^{-1} .
$$

Substitution of these expressions into Eq. (B8) yields

$$
\Phi_{2}\left(\boldsymbol{v}^{*}\right)=1+\left[b\left(v^{*}\right)-(1, b)\right]\left(\boldsymbol{v}^{*} \cdot \frac{\partial b}{\partial \boldsymbol{v}^{*}}, 1\right)^{-1} .
$$

The optimal choice for $b\left(v^{*}\right)$ would be that implying that $\Phi_{2}\left(\boldsymbol{v}^{*}\right)$ is an eigenfunction of $\mathcal{L}^{* \dagger}$ corresponding to the eigenvalue $\zeta_{0}^{*} / 2$. This is accomplished if $b\left(v^{*}\right)$ is the solution to

$$
\mathcal{L}^{* \dagger} b\left(v^{*}\right)=\frac{\zeta_{0}^{*}}{2}\left[b\left(v^{*}\right)+B^{-1}\right] .
$$

The solution to this equation, if it exists, has not yet been found.

\section{APPENDIX C: EVALUATION OF THE PERTURBATION THEORY RESULTS}

The expansion of the hydrodynamic eigenvalues of the linearized Boltzmann equation for small $k$ is given in Sec. V with the result

$$
\lambda_{i}(\boldsymbol{k})=\lambda_{i}(0)+k^{2} \lambda_{i}^{(2)}+\cdots,
$$

where

$$
\left\{\lambda_{i}(0)\right\}=\left\{0, \frac{\zeta_{0}^{*}}{2},-\frac{\zeta_{0}^{*}}{2}\right\},
$$

and

$$
\begin{aligned}
\lambda_{i}^{(2)}= & \left(\Phi_{i}, i \hat{\boldsymbol{k}} \cdot \boldsymbol{v}^{*} \Psi_{i}^{(1)}\right)+\left(\Phi_{i}, \mathcal{L}^{*} \mathcal{Q}_{i} \Psi_{i}^{(2)}\right) \\
= & \left(\Phi_{i}, \hat{\boldsymbol{k}} \cdot \boldsymbol{v}^{*}\left\{\mathcal{Q}_{i}\left[\mathcal{L}^{*}-\lambda_{i}(0)\right]\right\}^{-1} \mathcal{Q}_{i} \hat{\boldsymbol{k}} \cdot \boldsymbol{v}^{*} \Psi_{i}(0)\right) \\
& +\left(\Phi_{i}, \mathcal{L}^{*} \mathcal{Q}_{i} \Psi_{i}^{(2)}\right) .
\end{aligned}
$$

The eigenvalue $-\zeta_{0}^{*} / 2$ is $d$-fold degenerated and the convenient choice for the lowest order eigenfunctions has been discussed in Sec. IV. The formal expression for the second order eigenfunctions $\mathcal{Q}_{i} \Psi_{i}^{(2)}$ is given in Eq. (70). For $i \neq 2$, the second term on the right-hand side of Eq. (C3) vanishes since $\Phi_{i}$ is an eigenvector in those cases and, therefore,

$$
\left(\Phi_{i}, \mathcal{L}^{*} \mathcal{Q}_{i} \Psi_{i}^{(2)}\right)=\left(\mathcal{L}^{* \dagger} \Phi_{i}, \mathcal{Q}_{i} \Psi_{i}^{(2)}\right) \propto\left(\Phi_{i}, \mathcal{Q}_{i} \Psi_{i}^{(2)}\right)=0 .
$$

Then, Eq. (C3) can be rewritten as

$$
\begin{aligned}
\lambda_{i}^{(2)}= & \left(\hat{\boldsymbol{k}} \cdot \boldsymbol{v}^{*} \Phi_{i},\left\{\mathcal{Q}_{i}\left[\mathcal{L}^{*}-\lambda_{i}(0)\right]\right\}^{-1} \hat{\boldsymbol{k}} \cdot \boldsymbol{v}^{*} \Psi_{i}(0)\right) \\
& +\delta_{i, 2} \frac{1}{d}\left(\mathcal{L}^{* \dagger} v^{* 2}, \mathcal{Q}_{2} \Psi_{2}^{(2)}\right)
\end{aligned}
$$

where it has been used that

$$
\mathcal{P}_{i} \hat{\boldsymbol{k}} \cdot \boldsymbol{v}^{*} \Psi_{i}(0)=\Psi_{i}(0)\left(\Phi_{i} \hat{\boldsymbol{k}} \cdot \boldsymbol{v}^{*} \Psi_{i}(0)\right)=0 .
$$

This follows since $\Phi_{i}$ and $\Psi_{i}(0)$ have the same parity with respect to reflections of $\boldsymbol{v}^{*}$.

For the first eigenvalue it is

$$
\begin{aligned}
\lambda_{1}^{(2)} & =\left(\hat{\boldsymbol{k}} \cdot \boldsymbol{v}^{*},\left(\mathcal{Q}_{1} \mathcal{L}^{*}\right)^{-1} \hat{\boldsymbol{k}} \cdot \boldsymbol{v}^{*} \Psi_{i}(0)\right) \\
& =\left(\hat{\boldsymbol{k}} \cdot \boldsymbol{v}^{*}, \mathcal{L}^{*-1} \hat{\boldsymbol{k}} \cdot \boldsymbol{v}^{*} \Psi_{1}(0)\right)=\left(\mathcal{L}^{*+-1} \hat{\boldsymbol{k}} \cdot \boldsymbol{v}^{*}, \hat{\boldsymbol{k}} \cdot \boldsymbol{v}^{*} \Psi_{1}(0)\right) \\
& =-\frac{2}{\zeta_{0}^{*}}\left(\left(\hat{\boldsymbol{k}} \cdot \boldsymbol{v}^{*}\right)^{2}, \Psi_{1}(0)\right)=\frac{1}{\zeta_{0}^{*}} .
\end{aligned}
$$

In the first transformation, the property $\mathcal{P}_{1} \mathcal{L}^{*} X=0$, for arbitrary $X\left(v^{*}\right)$, has been employed. Next, consider the eigenvalue associated to the longitudinal component of $\boldsymbol{\Psi}_{3}(0)$ that we will denote by $\lambda_{\|}^{(2)}$,

$$
\begin{aligned}
\lambda_{\|}^{(2)}= & \left(\left(\hat{\boldsymbol{k}} \cdot \boldsymbol{v}^{*}\right)^{2},\left[\mathcal{Q}_{3}\left(\mathcal{L}^{*}+\frac{\zeta_{0}^{*}}{2}\right)\right]^{-1} \hat{\boldsymbol{k}} \cdot \boldsymbol{v}^{*} \hat{\boldsymbol{k}} \cdot \frac{\partial \ln \phi}{\partial \boldsymbol{v}^{*}}\right) \\
= & \left(v_{i}^{* 2},\left[\mathcal{Q}_{3}\left(\mathcal{L}^{*}+\frac{\zeta_{0}^{*}}{2}\right)\right]^{-1} G_{i i}^{*}\right)+\left(v_{i}^{* 2},\left[\mathcal{Q}_{3}\left(\mathcal{L}^{*}+\frac{\zeta_{0}^{*}}{2}\right)\right]^{-1}\right. \\
& \left.\times\left[\Psi_{1}(0)+\frac{d+1}{d} \Psi_{2}(0)\right]\right)=\left(v_{i}^{* 2},\left(\mathcal{L}^{*}+\frac{\zeta_{0}^{*}}{2}\right)^{-1} G_{i i}^{*}\right) \\
& +\frac{2}{\zeta_{0}^{*}}\left(v_{i}^{* 2}, \Psi_{1}(0)\right)+\frac{d-1}{d} \frac{1}{\zeta_{0}^{*}}\left(v_{i}^{* 2}, \Psi_{2}(0)\right),
\end{aligned}
$$

where it has been taken into account that $\mathcal{P}_{3}\left(\mathcal{L}^{*}+\zeta_{0}^{*} / 2\right)=0$. Using Eq. (A19) in the first term on the right-hand side of the above equation and evaluating explicitly the other two ones one gets 


$$
\lambda_{\|}^{0}=\left(v_{i}^{* 2}, C_{i i}^{*}\right)+\frac{1}{d \zeta_{0}^{*}}=\frac{2(d-1)}{d} \eta^{*}+\frac{1}{d \zeta_{0}^{*}} .
$$

The last equality follows from the fact that the expression of $\eta^{*}$ given in Eq. (A15) is equivalent to

$$
\left(D_{i j}^{*}, C_{i j}^{*}\right)=\eta^{*}\left(1+\frac{d-2}{d} \delta_{i, j}\right),
$$

because of the symmetry of the tensors $\mathrm{D}$ and $\mathrm{C}$. The calculation of the shear modes eigenvalues $\lambda_{\perp}^{(2)}$ is straightforward:

$$
\begin{aligned}
\lambda_{\perp}^{(2)} & =\left(v_{\|}^{*} v_{\perp i}^{*},\left(\mathcal{L}^{*}+\frac{\zeta_{0}^{*}}{2}\right)^{-1} v_{\|}^{*} \Psi_{3, \perp i}\right) \\
& =\left(D_{\|, \perp i}^{*},\left(\mathcal{L}^{*}+\frac{\xi_{0}^{*}}{2}\right)^{-1} G_{\|, \perp i}^{*}\right) \\
& =\left(D_{\|, \perp i}^{*}, C_{\|, \perp i}^{*}\right)=\eta^{*} .
\end{aligned}
$$

Finally, the evaluation of $\lambda_{2}^{(2)}$ is somewhat more complicated. The contributions from each of the two terms in Eq. (C5) will be computed separately. The first one is given by

$$
\begin{gathered}
\left(\hat{\boldsymbol{k}} \cdot \boldsymbol{v}^{*} \Phi_{2},\left[\mathcal{Q}_{2}\left(\mathcal{L}^{*}-\frac{\zeta_{0}^{*}}{2}\right)\right]^{-1} \hat{\boldsymbol{k}} \cdot \boldsymbol{v}^{*} \Psi_{2}(0)\right) \\
=\left(v_{i}^{*} \Phi_{2},\left(\mathcal{L}-\frac{\zeta_{0}^{*}}{2}\right)^{-1} v_{i}^{*} \Psi_{2}(0)\right),
\end{gathered}
$$

that is equivalent to

$$
\begin{aligned}
& \frac{1}{d}\left(S_{i}^{*},\left(\mathcal{L}-\frac{\zeta_{0}^{*}}{2}\right)^{-1} v_{i}^{*} \Psi_{2}(0)\right) \\
& \quad+\frac{d-1}{d}\left(v_{i}^{*},\left(\mathcal{L}-\frac{\zeta_{0}^{*}}{2}\right)^{-1} v_{i}^{*} \Psi_{2}(0)\right) \\
& =\frac{1}{d}\left(S_{i}^{*}, \mathcal{A}_{i}^{*}\right)-\frac{2}{d \zeta_{0}^{*}}\left(S_{i}^{*}, \Psi_{3, i}(0)\right)-\frac{d+1}{d} \frac{1}{\zeta_{0}^{*}}\left(v_{i}^{*}, v_{i}^{*} \Psi_{2}(0)\right) \\
& =\kappa^{*}-\frac{d+1}{d \zeta_{0}^{*}} .
\end{aligned}
$$

The analysis of the second term on the right-hand side of Eq. (C5) is carried out in an analogous way,

$$
\frac{1}{d}\left(\mathcal{L}^{*} v^{* 2}, \mathcal{Q}_{2} \Psi_{2}^{(2)}\right)=-\frac{\zeta_{0}^{*}}{d}\left(v^{* 2}, \mathcal{Q}_{2} \Psi_{2}^{(2)}\right)+\frac{1}{d}\left(a, \mathcal{Q}_{2} \Psi_{2}^{(2)}\right)
$$

It is

$$
\begin{aligned}
-\frac{\zeta_{0}^{*}}{d}\left(v^{* 2}, \mathcal{Q}_{2}^{2} \Psi_{2}^{(2)}\right) & =\frac{\zeta_{0}^{*}}{2}\left(\Phi_{1}, \mathcal{Q}_{2} \Psi_{2}^{(2)}\right) \\
& =-\frac{1}{\zeta_{0}^{*}}\left(\Phi_{3, i}, v_{i}^{*} \Psi_{2}(0)\right)=-\frac{1}{\zeta_{0}^{*}}
\end{aligned}
$$

In the second equality above, the explicit expression of $\Psi_{2}^{(2)}$ given in Eq. (70) as well as the properties of $\mathcal{L}^{*}$ have been used. The second term on the right-hand side of Eq. (C14) can be rewritten as

$$
\frac{1}{d}\left(a, \mathcal{Q}_{2} \psi_{2}^{(2)}\right)=-\frac{1}{d}\left(a, \mathcal{M}_{1}^{*}\right),
$$

where $\mathcal{M}_{1}^{*}$ is defined in Eq. (A29). Substitution of Eqs. (C15) and (C16) into Eq. (C14) yields

$$
\frac{1}{d}\left(\mathcal{L}^{*} v^{* 2}, \mathcal{Q}_{2} \Psi_{2}^{(2)}\right)=-\frac{1}{\zeta_{0}^{*}}-\frac{1}{d}\left(a, \mathcal{M}_{1}^{*}\right)=-\zeta_{1}^{*},
$$

because of Eq. (A28). Then, use of Eqs. (C13) and (C17) into Eq. (C5) gives the final expression for $\lambda_{2}^{(2)}$,

$$
\lambda_{2}^{(2)}=\kappa^{*}-\zeta_{1}^{*}-\frac{d+1}{d \zeta_{0}^{*}} .
$$

Comparison of the results for the second order eigenvalues obtained in this Appendix with those reported in Sec. II shows that both agree, with the transport coefficients given by the same expressions in both cases.

\section{APPENDIX D: LINEARIZATION OF THE MODEL KINETIC EQUATION}

Solutions to the model kinetic equation (72) are sought of the form (32) and, consistently, $g=g_{\mathrm{HCS}}+\delta g$ and $\nu=\nu_{\mathrm{HCS}}$ $+\delta \nu$, retaining only terms linear in $\Delta$,

$$
\begin{aligned}
\left(\frac{\partial}{\partial t}+\boldsymbol{v} \cdot \boldsymbol{\nabla}\right)\left(f_{\mathrm{HCS}} \Delta\right)= & -\delta \nu\left(f_{\mathrm{HCS}}-g_{\mathrm{HCS}}\right)-\nu_{\mathrm{HCS}}\left(f_{\mathrm{HCS}} \Delta\right. \\
& -\delta g) .
\end{aligned}
$$

Use has been made of the HCS model equation

$$
\frac{\partial f_{\mathrm{HCS}}}{\partial t}=\frac{\zeta_{\mathrm{HCS}}}{2} \frac{\partial}{\partial \boldsymbol{v}} \cdot\left(\boldsymbol{v} f_{\mathrm{HCS}}\right)=-\nu_{\mathrm{HCS}}\left(f_{\mathrm{HCS}}-g_{\mathrm{HCS}}\right) .
$$

Next, from the choice of $\nu$ as corresponding to hard sphere behavior,

$$
\delta \nu=\nu_{\mathrm{HCS}}\left(\frac{\delta n}{n_{\mathrm{HCS}}}+\frac{2 \delta T}{T_{\mathrm{HCS}}}\right) .
$$

Similarly, the definition of $g$ in Eq. (73) gives

$$
\delta g=\frac{g_{\mathrm{HCS}}}{n_{\mathrm{HCS}}} \delta n+\frac{\partial g_{\mathrm{HCS}}}{\partial T_{\mathrm{HCS}}} \delta T-\frac{\partial g_{\mathrm{HCS}}}{\partial \boldsymbol{v}} \cdot \boldsymbol{u} .
$$

The linear model kinetic equation (D1) then becomes

$$
\begin{aligned}
\left(\frac{\partial}{\partial t}+\boldsymbol{v} \cdot \boldsymbol{\nabla}\right)\left(f_{\mathrm{HCS}} \Delta\right)= & -\left(\frac{\delta n}{n_{\mathrm{HCS}}}+\frac{\delta T}{2 T_{\mathrm{HCS}}}\right) \nu_{\mathrm{HCS}}\left(f_{\mathrm{HCS}}-g_{\mathrm{HCS}}\right) \\
& -\nu_{\mathrm{HCS}}\left(f_{\mathrm{HCS}} \Delta-\frac{g_{\mathrm{HCS}}}{n_{\mathrm{HCS}}} \delta n-\frac{\partial g_{\mathrm{HCS}}}{\partial T_{\mathrm{HCS}}} \delta T\right. \\
& \left.+\frac{\partial g_{\mathrm{HCS}}}{\partial \boldsymbol{v}} \cdot \boldsymbol{u}\right) .
\end{aligned}
$$

It is convenient to eliminate $g_{\mathrm{HCS}}$ in this result using the HCS equation (D2) to get 


$$
\begin{aligned}
\left(\frac{\partial}{\partial t}\right. & +\boldsymbol{v} \cdot \boldsymbol{\nabla})\left(f_{\mathrm{HCS}} \Delta\right)=-\nu_{\mathrm{HCS}} f_{\mathrm{HCS}} \Delta+\left(\frac{\delta n}{n_{\mathrm{HCS}}}\right. \\
& \left.+\frac{\delta T}{2 T_{\mathrm{HCS}}}\right) \frac{\zeta_{\mathrm{HCS}}}{2} \frac{\partial}{\partial \boldsymbol{v}} \cdot\left(\boldsymbol{v} f_{\mathrm{HCS}}\right)+\frac{\delta n}{n_{\mathrm{HCS}}}\left[\nu_{\mathrm{HCS}} f_{\mathrm{HCS}}\right. \\
& \left.+\frac{\zeta_{\mathrm{HCS}}}{2} \frac{\partial}{\partial \boldsymbol{v}} \cdot\left(\boldsymbol{v} f_{\mathrm{HCS}}\right)\right] \\
& +\delta T\left[\nu_{\mathrm{HCS}} \frac{\partial f_{\mathrm{HCS}}}{\partial T_{\mathrm{HCS}}}+\frac{\zeta_{\mathrm{HCS}}}{2} \frac{\partial}{\partial \boldsymbol{v}} \cdot\left(\boldsymbol{v} \frac{\partial f_{\mathrm{HCS}}}{\partial T_{\mathrm{HCS}}}\right)\right] \\
& -\boldsymbol{u} \cdot \frac{\partial}{\partial \boldsymbol{v}}\left[\nu_{\mathrm{HCS}} f_{\mathrm{HCS}}+\frac{\zeta_{\mathrm{HCS}}}{2} \frac{\partial}{\partial \boldsymbol{v}} \cdot\left(\boldsymbol{v} f_{\mathrm{HCS}}\right)\right]
\end{aligned}
$$

The right-hand side of this expression can be put in a more transparent form by noting that

$$
\left\{\left(\Delta, \Phi_{i}\right)\right\}=\left\{\frac{\delta n}{n_{\mathrm{HCS}}}, \frac{\delta T}{2 T_{\mathrm{HCS}}}+\frac{\delta n}{n_{\mathrm{HCS}}}, \frac{u_{\|}}{v_{\mathrm{HCS}}}, \frac{\boldsymbol{u}_{\perp}}{v_{\mathrm{HCS}}}\right\} .
$$

where the scalar product $(X, Y)$ is defined in Eq. (40) and $\left\{\Phi_{i}\right\}$ are the biorthogonal set given in Eq. (58). Moreover, the relation

$$
T_{\mathrm{HCS}} \frac{\partial \ln f_{\mathrm{HCS}}}{\partial T_{\mathrm{HCS}}}=\frac{1}{2 \phi} \frac{\partial}{\partial \boldsymbol{v}} \cdot(\boldsymbol{v} \phi),
$$

allows one to write the dependence on $f_{\mathrm{HCS}}$ on the right-hand side in terms of the lowest order hydrodynamic eigenfunctions $\left\{\Psi_{i}(0)\right\}$ of the linearized Boltzmann operator given in Eq. (54). Then, Eq. (D6) can be rewritten as

$$
\begin{aligned}
\left(\frac{\partial}{\partial t}+\boldsymbol{v} \cdot \boldsymbol{\nabla}\right)\left(f_{\mathrm{HCS}} \Delta\right)= & -\nu_{\mathrm{HCS}} f_{\mathrm{HCS}}\left[\Delta-\sum_{i} \Psi_{i}(0)\left(\Phi_{i}, \Delta\right)\right] \\
& +\frac{\zeta_{\mathrm{HCS}}}{2} \frac{\partial}{\partial \boldsymbol{v}} \cdot\left[f_{\mathrm{HCS}} \boldsymbol{v} \sum_{i} \Psi_{i}(0)\left(\Phi_{i}, \Delta\right)\right] \\
& -f_{\mathrm{HCS}} \frac{v_{\mathrm{HCS}}(t)}{\ell} \sum_{i} \lambda_{i}(0) \Psi_{i}(0)\left(\Phi_{i}, \Delta\right) .
\end{aligned}
$$

Here $\left\{\lambda_{i}(0)\right\}$ are the eigenvalues of Eq. (53).

Finally, introducing the dimensionless variables of Secs. II and III, and the single Fourier component of Eq. (35) gives the linear kinetic equation for the model

$$
\left(\partial_{s}+\boldsymbol{i} \boldsymbol{k} \cdot \mathbf{v}^{*}+\mathcal{L}_{m}^{*}\right) \widetilde{\Delta}\left(\boldsymbol{k}, \boldsymbol{v}^{*}, s\right)=0,
$$

with

$$
\mathcal{L}_{m}^{*}=\sum_{i} \lambda_{i}(0) \mathcal{P}_{i}+\nu_{0}^{*} \mathcal{Q}+\frac{\zeta_{0}^{*}}{2} \phi^{-1} \frac{\partial}{\partial \boldsymbol{v}^{*}} \cdot \boldsymbol{v}^{*} \phi \mathcal{Q} .
$$

The projection operators $\mathcal{P}_{i}$ are defined in Eq. (62), and

$$
\mathcal{P} \equiv \sum_{i} \mathcal{P}_{i}, \quad \mathcal{Q} \equiv 1-\mathcal{P} .
$$

It is directly verified that

$$
\mathcal{P} \phi^{-1} \frac{\partial}{\partial \boldsymbol{v}^{*}} \cdot \boldsymbol{v}^{*} \phi \mathcal{Q}=0,
$$

so that $\mathcal{L}_{m}^{*}$ can be written

$$
\mathcal{L}_{m}^{*}=\sum_{i} \lambda_{i}(0) \mathcal{P}_{i}+\mathcal{Q}\left[\nu_{0}^{*}+\frac{\zeta_{0}^{*}}{2} \phi^{-1} \frac{\partial}{\partial \boldsymbol{v}^{*}} \cdot \boldsymbol{v}^{*} \phi\right] \mathcal{Q} .
$$

This is the expression for the generator of the linear dynamics used in main the text.
[1] P. K. Haff, J. Fluid Mech. 134, 401 (1983).

[2] J. T. Jenkins and M. W. Richman, Arch. Ration. Mech. Anal. 87, 355 (1985).

[3] C. K. W. Lun, S. B. Savage, D. J. Jeffrey, and N. Chepurnuy, J. Fluid Mech. 140, 223 (1984).

[4] A. Goldshtein and M. Shapiro, J. Fluid Mech. 282, 41, (1995).

[5] J. J. Brey, J. W. Dufty, C. S. Kim, and A. Santos, Phys. Rev. E 58, 4638 (1998).

[6] J. J. Brey and D. Cubero, in Granular Gases, edited by T. Pöschel and S. Luding (Springer-Verlag, Berlin, 2001).

[7] J. J. Brey, M. J. Ruiz-Montero, and D. Cubero, Europhys. Lett. 48, 359 (1999); J. J. Brey, M. J. Ruiz-Montero, and F. Moreno, Phys. Rev. E 62, 5339 (2000).

[8] I. Goldhirsch, Annu. Rev. Fluid Mech. 35, 267 (2003).

[9] J. J. Brey and M. J. Ruiz-Montero, Europhys. Lett. 66, 805 (2004).

[10] J. A. McLennan, Introduction to Nonequilibrium Statistical Mechanics (Prentice-Hall, New Jersey, 1989).
[11] G. Scharf, Helv. Phys. Acta 40, 929 (1967); 42, 5 (1969).

[12] J. W. Dufty and J. J. Brey, Phys. Rev. E 68, 030302(R) (2003); J. J. Brey, J. W. Dufty, and M. J. Ruiz-Montero in Granular Gas Dynamics, edited by T. Pöschel and N. Brilliantov (Springer-Verlag, Berlin 2003).

[13] J. J. Brey, J. W. Dufty, and A. Santos, J. Stat. Phys. 97, 281322, (1999).

[14] T. P. C. van Noije and M. H. Ernst, in Granular Gases, edited by T. Pöschel and S. Luding (Springer-Verlag, Berlin, 2001); J. W. Dufty, Adv. Complex Syst. 4, 397 (2001).

[15] N. K. Brilliantov and T. Pöschel, Kinetic Theory of Granular Gases (Oxford University Press, Oxford, 2004).

[16] J. J. Brey, M. J. Ruiz-Montero, and D. Cubero, Phys. Rev. E 54, 3664 (1996).

[17] G. Bird, Molecular Gas Dynamics and the Direct Simulation of Gas Flows (Clarendon Press, Oxford, 1994); A. L. García, Numerical Methods for Physics (Prentice-Hall, Englewood Cliffs, NJ, 2000). 
[18] N. Dunford and J. Schwartz, Linear Operators (Interscience Publishers, New York, 1967).

[19] J. J. Brey, F. Moreno, and J. W. Dufty, Phys. Rev. E 54 ,445 (1996).

[20] C. Cercignani, Theory and Applications of the Boltzmann
Equation (Elsevier, New York, 1975).

[21] J. W. Dufty, A. Baskaran, and L. Zogaib, Phys. Rev. E 69, 051301 (2004); A. Baskaran and J. W. Dufty, cond-mat/ 0410084. 